\title{
A Quarter Century of Pharmacognostic Research on Panamanian Flora: A Review*
}

\author{
Authors \\ Catherina Caballero-George ${ }^{1}$, Mahabir P. Gupta ${ }^{2}$ \\ Affiliations \\ ${ }^{1}$ Institute of Scientific Research and High Technology Services (INDICASAT-AIP), Panama, Republic of Panama \\ ${ }^{2}$ Center for Pharmacognostic Research on Panamanian Flora (CIFLORPAN), College of Pharmacy, University of Panama, \\ Panama, Republic of Panama
}

\section{Key words \\ - bioassays \\ - Panamanian plants \\ - ethnomedicine \\ - novel compounds}

received March 25, 2011

revised May 5, 2011

accepted May 10, 2011

Bibliography

Dol http://dx.doi.org/

10.1055/s-0030-1271187

Published online June 14, 2011

Planta Med 2011; 77:

1189-1202 @ Georg Thieme

Verlag KG Stuttgart · New York

ISSN 0032-0943

\section{Correspondence}

Prof. Dr. Mahabir P. Gupta

Center for Pharmacognostic

Research on Panamanian Flora

(CIFLORPAN)

College of Pharmacy

Box 0824-00172

Panama

Republic of Panama

Phone: + 5075236311

Fax: + 5072640789

mahabirpgupta@gmail.com

\section{Abstract}

$\nabla$

Panama is a unique terrestrial bridge of extreme biological importance. It is one of the "hot spots" and occupies the fourth place among the 25 most plant-rich countries in the world, with $13.4 \%$ endemic species. Panamanian plants have been screened for a wide range of biological activities: as cytotoxic, brine shrimp-toxic, antiplasmodial, antimicrobial, antiviral, antioxidant, immunosuppressive, and antihypertensive agents. This review concentrates on ethnopharmacological uses of medicinal plants employed by three Amerindian groups of Panama and on selected plants

\section{Introduction}

\section{$\nabla$}

Medicinal plants remain an endless source of new drugs, new drug leads, and new chemical entities (NCEs) [1]. In developing countries, medicinal plants have been the most accessible source of medicaments and in rural areas, traditional medicine is part of the first line of treatment for common pathologies [2]. The geographical characteristics of the Isthmus of Panama are responsible for its highly diverse flora and fauna. This diversity promotes the use of plants in traditional medicine by the Amerindian groups and the rural population, which have limited access to modern drugs. Panama is a unique terrestrial bridge of great biological importance. Panamanian flora is one of the richest in the world with an estimate of 9893 different species of vascular plants, of which 1327 (13.4\%) are endemic [3]. There is a large number of nonvascular species but they have not been investigated fully. Panama has over 687 species of ferns and about 1000 species of orchids, of which $50 \%$ are endemic. Epiphytes, lianas, and climbers

\footnotetext{
* Invited review - Planta Medica special supplement;
} editor, Prof. Dr. H. Kolodziej. with novel structures and/or interesting bioactive compounds. During the last quarter century, a total of approximately 390 compounds from 86 plants have been isolated, of which 160 are new to the literature. Most of the work reported here has been the result of many international collaborative efforts with scientists worldwide. From the results presented, it is immediately obvious that the Panamanian flora is still an untapped source of new bioactive compounds.

Supporting information available at http://www.thieme-connect.de/ejournals/toc/ plantamedica

are a major component of the Panamanian tropical forest. Mosses abound in moist cloud forests as well as other parts of the country. Panama is considered one of the botanical "hot spot" countries and occupies the fourth place in the world in number of vascular species [4]. The number of species per $10000 \mathrm{~km}^{2}$ ranks even much higher than in bigger countries like India, China, and the United States [5].

Evidence of the biomedical potential of herbal drugs used in Panamanian traditional medicine is continually being reported. Panamanian plants have been evaluated for their biological activities on a diverse range of disease targets. They have been studied inter alia as cytotoxic to cancer cell lines, antimalarial, antileishmanial, antimicrobial, antiviral, larvicidal, antioxidant, immunosupressive, acetylcholinesterase inhibitors, and antihypertensive agents [6-10].

Research on the highly diverse Panamanian flora proposes new economical and medicinal ventures attractive to the pharmaceutical industry. In the National Science Strategic Plan 2010-2014, a very high priority has been assigned to the study and utilization of Panamanian biodiversity as a source 
of molecules for the agrochemical, pharmaceutical, food, and cosmetic industries [11].

This paper summarizes and updates two earlier reviews $[6,7]$ on the current available knowledge on Panamanian medicinal plants and the numerous investigations carried out during the last quarter century on their biomedical potential, resulting in identification of compounds with new chemical structures and possible new therapeutic and/or other applications.

\section{Ethnomedical Surveys and Traditional Medicine $\nabla$}

Systematic studies on Panamanian flora were initially based on selecting plants for phytochemical and pharmacological studies based on ethnopharmacological uses. In Panama, the Amerindian groups and the rural population have depended on plant preparations to treat a range of ailments, and quite remarkably with a great degree of success [12]. Panama is home to five Amerindian groups: Ngöbe-Buglé, Kuna, Emberá-Wounan, Naso or Teribe, and Bri-Bri, representing 9.6\% of the total population [13]. These ethnopharmacological surveys collected a large amount of data from three major groups in Panama namely the Kunas, the Ngöbe-Buglé, and the Teribes.

Among the indigenous groups of Latin America, the Kunas have been able to preserve their cultural and political autonomy by means of a robust social organization. In spite of the Panamanian government support in health care, this ethnic group relies on the use of medicinal plants to treat a variety of illnesses and to perform religious rituals. The most common health problems in this community are malnutrition, tuberculosis, pneumonia, malign tumors in conditions like albinism, and intestinal problems. From the 90 species of plants employed in their medicine and rituals ( $\odot$ Table 1), 49 plants were used topically to treat skin and eyes infections and as wound healing, 24 were used internally to treat fever, pain, cold, and general weakness, and 17 plants were used in both routes. Interestingly, $19 \%$ of the plants used in Kuna's traditional medicine were related to childbirth [14].

The Ngöbe-Buglé is the largest indigenous group in Panama (65\% of the total indigenous population) [13]. During different field trips in the homeland of the Ngöbe-Buglé since the late eighties, a total of 104 medicinal plants have been collected and identified (O Table 1) $[15,16]$.

The Teribes represent the third most important group of Amerindians in the country. The use of medicinal plants is very common in this group, and the passing of this knowledge is the responsibility of the elders. The most common applications for medicinal plants were in the treatment of colds, headache, body aches, vertigo, cramps, diarrhea, furunculosis, leishmaniasis, and snakebites. From this study, 108 plants were identified ( $\bullet$ Table 1) [17].

\section{Access Rights and Legal Framework}

$\nabla$

Panama values international cooperation and is aware of the need of increased international funding and specific initiatives to support research into priority areas to investigate the potential of unexplored Panamanian biodiversity.

As signatory to the Convention of the Biological Diversity (CBD), Panama encourages the objectives of the Convention: conservation of biological diversity, sustainable use of its components, and the fair and equitable sharing of the benefits arising out of the utilization of genetic resources [18], and it is aware that intel- lectual property rights "may have an influence on the implementation" of the CBD and that it is compulsory for states to cooperate in order to ensure that intellectual property rights are "supportive of and do not run counter to" the objectives of the CBD [19].

Panama has put in place legislation through the Executive Decree 257 of 17 October 2006 and regulations of Article 71 of the General Law of Environment. Recently the Executive Decree No. 25 dated 29 of April 2009 has updated the legislation and now governs the regulations concerning access to the genetic resources of Panama. For this purpose a special unit, "Unidad Nacional de Recursos Genéticos" (UNARGEN), under the supervision of the National Environmental Authority has been created [20]. According to these regulations, collection and export permits issued by UNARGEN are required for research within the country and for sending samples out of Panama. Clear distinction is made between fundamental research and bioprospection studies. Material transfer agreements have to be signed by the collaborative scientists. Provisions are set forth for indigenous rights, equitable sharing of profits, and sovereignty rights of Panama on plant material and for conservation of biodiversity. This facilitates the exchange of samples and international collaboration while at the same time complying with the CBD.

\section{Screenings of Panamanian Plants \\ $\nabla$}

It has been estimated that from a total of 300000 identified plant species worldwide, only $6 \%$ of this total has been pharmacologically studied and only $15 \%$ phytochemically [21].

Four approaches were used to screen Panamanian plants for further phytochemical research. Plant selection was based on chemotaxonomy, ethnopharmacological information, and random selection of plants within an area of 0.1 ha biodiversity plots in national parks and ecologically-based bioprospection.

Selected Panamanian plant extracts were subjected to a range of biological screens. They were tested for toxicity, in particular against Artemia salina [22] and cancer cell lines [23], as antimicrobial [24], antifungal [25], molluscicidal [6], and DNA intercalation [26] agents, as inhibitors of protease and reverse transcriptase [27], for antiplasmodial [28], anti-inflammatory [29,30], antihyperglycemic [31], and anti-HSV1 [27] activities, for toxicity against Aedes aegypti [31], and as inhibitors of receptor-binding assays on endothelin $\mathrm{ET}_{\mathrm{A}}$, angiotensin $\mathrm{AT}_{1}$, and neuropeptide $\mathrm{Y}$ $\mathrm{Y}_{1}$ receptors [10].

\section{Isolation of Compounds from Panamanian Plants $\nabla$}

- Table 2 summarizes the chemical composition and corresponding biological activities of selected Panamanian plants. Phytochemical research on candidate plants chosen after biological and chemical screening has led to the isolation of numerous new compounds, many of which have a wide array of valuable biological activities. This review has concentrated on selected plants with novel structures and/or interesting bioactive compounds. O Table 2 summarizes in alphabetical order of the families and plants, novel and known compounds isolated, and the biological activity attributed to one or more of the compounds. This table was constructed from original publications of our group and other Panamanian scientists. The most important plant families which yielded active compounds were Clusaceae, Boragina- 
Table 1 Medicinal plant inventory of Kuna, Ngöbe-Buglé, and Teribe Indians.

\begin{tabular}{|c|c|c|}
\hline Family & Specie & Application (plant part; preparation; Amerindian group) \\
\hline \multirow[t]{16}{*}{ Acanthaceae } & Aphelandra hartwegiana Nees & Constipation (F; I; K), antiemetic (WP; D; NB) \\
\hline & Aphelandra aurantiaca (Scheidw.) & Before childbirth (WP; I; NB) \\
\hline & Aphelandra sinclairiana Nees & Antiemetic in pregnancy (St; D; NB) \\
\hline & Aphelandra tonduzii Leonard. & Fever, skin rash, mumps, erysipelas (WP; D; NB) \\
\hline & Blechum costaricense Oerst. & Vertigo (L; D; TB) \\
\hline & Blechum panamense Lindau & Vertigo (St, L; D; TB) \\
\hline & Blechum pyramidatum (Lam.) Urb. & Antiemetic (WP; D; NB), amebiasis (L; D; TB) \\
\hline & Justicia sp. & Internal fever (WP; I; K) \\
\hline & Justicia ephemera Leonard & Convulsions (WP; ID; K) \\
\hline & Justicia oerstedii Leonard. & Diarrhea (St, R; D; TB) \\
\hline & Justicia pectoralis Jacq. & Pain, hepatic disorders (St; I; NB) \\
\hline & Sanchezia pennellii Leonard & "Enlargement of the mind" $(\mathrm{F} ; \mathrm{I} ; \mathrm{K})$ \\
\hline & Odontonema tubiforme (Bertol.) Kuntze & Anti-inflammatory (L; D; NB), induce child birth (St, L; D; TB) \\
\hline & Ruellia biolleyi Lindau. & Dizziness, edema, fever (St; D; NB), appetite stimulant (St, L; D; TB) \\
\hline & Ruellia cf. metallica Leonard & Body ache, fever (St; D; TB) \\
\hline & Ruellia praeclara Standl. & Edema (St; D; NB) \\
\hline \multirow[t]{2}{*}{ Amaranthaceae } & Amaranthus sp. & Antiemetic, hematuria (L, F; I; K) \\
\hline & Pleuropetalum sprucei (Hook. f.) Standl. & Nose bleeding, post-measles weakness (St; D; TB) \\
\hline Amaryllidaceae & Crinum darienensis Woodson & Fever (WP; I; K) \\
\hline \multirow[t]{3}{*}{ Annonaceae } & Annona reticulata $\mathrm{L}$. & Inflammation, fractures (L; C; TB) \\
\hline & Cymbopetalum costarricense (J.D. Sm.) R. E. Fries. & Snakebite (B; I; NB) \\
\hline & Xylopia frutescens Aubl. & Anthelmintic, antipyretic (L; NS; NB) \\
\hline \multirow[t]{4}{*}{ Apocynaceae } & Odontadenia puncticulosa (L. Rich.) Pulle. & Rubella (WP; I; NB) \\
\hline & Peltastes colombianus Woods. & Diarrhea (St; I; NB) \\
\hline & Rhabdadenia biflora (Jacq.) Muell.-Arg. & Eye infections ( $\mathrm{F} ; \mathrm{l} ; \mathrm{K})$ \\
\hline & Stemmadenia grandiflora (Jacq.) Miers & Renal disorders (St; I; NB) \\
\hline \multirow[t]{15}{*}{ Araceae } & Anthurium sp. & To reduce size of the uterus during pregnancy $(F ; l ; K)$, antidiarrhoeic (R; D; NB) \\
\hline & Anthurium pentaphyllum (Aubl.) G. Don. & Skin infections, pain reliever, inflammation of muscles and joints (WP; D; K) \\
\hline & Dieffenbachia aurantiaca Engl. & Pain reliever, skin infections (WP; D; K) \\
\hline & Dracontium costaricense Engler. & Snakebite (L; D; NB) \\
\hline & Dracontium dressleri Croat. & To facilitate childbirth (F; IK; K), muscle aches, snakebite (L, R; NS; K) \\
\hline & Dracontium spruceanum (Schott) G. H. Zhu & Snakebite (Rz, St, T; D; TB), skin ulcers (T; D; TB) \\
\hline & Homalomena wendlandii Schott & Snakebite (St; D; TB) \\
\hline & Montrichardia arborescens (L.) Schott. & Skin infections (L; D; K) \\
\hline & Philodendron sp. & To increase vitality in children (WP; I; K), wound healing (S; DA; K), skin rash, fever (WP; D; NB) \\
\hline & Philodendron radiatum Schott & Pain, snakebite (R; C; TB) \\
\hline & Spathiphyllum friedrichsthalii Schott. & To avoid enlargement of the uterus during pregnancy, to facilitate childbirth $(\mathrm{F} ; \mathrm{I} ; \mathrm{K})$ \\
\hline & Spathiphyllum quindiuense Engl. & To bathe sick children (WP; I; K) \\
\hline & Xanthosoma sp. & Strong muscular and bone aches, inflammation (In; D; K) \\
\hline & Xanthosoma helleborifolium (Jacq.) Shott. & To facilitate childbirth (T; IK; K) \\
\hline & Xanthosoma mexicanum Liebm. & To facilitate childbirth, snakebite (F; ; K) \\
\hline \multirow[t]{6}{*}{ Aristolochiaceae } & Aristolochia aff. grandiflora Sw. & Snakebite (L; D; TB) \\
\hline & Aristolochia chapmaniana Stand. & Snakebite, shortness of breath (WP; I; NB) \\
\hline & Aristolochia constricta Griseb. & Snakebite (WP; I; NB), (St; D; TB); leishmaniasis (L; C; TB) \\
\hline & Aristolochia pilosa H. B.K. & Snakebite (WP; I; NB) \\
\hline & Aristolochia sylvicola Stand. & Snakebite (St; D; NB) \\
\hline & Aristolochia tonduzii O.C. Schmidt & Pain, inflammation (St; D; TB) \\
\hline Asclepiadaceae & Sarcostemma clausum (Jacq.) Roem. et Schult. & Shortness of breath (St; I; NB) \\
\hline Aspleniaceae & Asplenium serratum L. & Burns (Sc; C; TB) \\
\hline \multirow[t]{8}{*}{ Asteraceae } & Bidens reptans (L.) G. Don. & Sore eyes (St; D; NB) \\
\hline & Hebeclinium macrophyllum (L.) DC. & Insect bites (L; D; TB) \\
\hline & Neurolaena lobata (L.) R. Br. & Skin infections (WP; D; K), (L;D;TB); fever (St; I; NB) \\
\hline & Sinclairia polyantha (Klatt) Rydb. & Cough (St; D; TB) \\
\hline & Sphagneticola trilobata (L.) Pruski & Cough, flu (L; D; TB) \\
\hline & Rolandra fruticosa (L.) Kuntze & Muscle aches (WP; D; K) \\
\hline & Vernonia sp & To facilitate expulsion of the placenta (In; I; K) \\
\hline & Wedelia trilobata (L.) Hitchc. & Skin diseases (WP; D; K) \\
\hline \multirow[t]{4}{*}{ Begoniaceae } & Begonia glabra Aubl. & Furuncles (WP; fresh; NB) \\
\hline & Begonia hirsuta Aubl. & Renal disorders (WP; D; NB) \\
\hline & Begonia multinervia Liebm. & Fever (Pt; Jc; TB) \\
\hline & Begonia semiovata Liebm. & Women's ailments (WP; D; NB), chicken pox (St; D; TB) \\
\hline \multirow[t]{2}{*}{ Bignoniaceae } & Arrabidaea verrucosa (Standl.) A. Gentry & Fever, weakness, muscle aches (WP; I; K) \\
\hline & Callichlamys latifolia (L.C. Rich) K. Schum. & Body aches, loss of appetite (St; NS; NB) \\
\hline
\end{tabular}


Table 1 Medicinal plant inventory of Kuna, Ngöbe-Buglé, and Teribe Indians. (continued)

\begin{tabular}{|c|c|c|}
\hline \multirow[t]{4}{*}{ Family } & Specie & Application (plant part; preparation; Amerindian group) \\
\hline & Crescentia cujete L. & Purgative (F; $\mathrm{D} ; \mathrm{TB})$ \\
\hline & Mansoa standleyi (Steyerm.) A. H. Gentry & Aggressive dementia (VI; D; TB) \\
\hline & Stizophyllum riparium (HBK.) Sandw. & Anuria (WP; D; K), urinary infections (St; D; TB) \\
\hline Blechnaceae & Salpichlaena volubilis (Kaulf.) J. Sm. & Toothache (VI; D; TB) \\
\hline \multirow[t]{4}{*}{ Boraginaceae } & Cordia alliodora (Ruiz \& Pav.) Oken & Fever (L; D; TB) \\
\hline & Cordia spinescens $\mathrm{L}$. & Wound healing (St; DA; NB) \\
\hline & Tournefortia bicolor Sw. & Fever (St, L; D; TB) \\
\hline & Tournefortia cuspidata Kunth & Fever (St; D; TB) \\
\hline \multirow[t]{2}{*}{ Burseraceae } & Bursera simaruba (L.) Sarg. & Urinary infections, bruises, contraconceptive (St; D; TB) \\
\hline & Trattinnickia aspera (Standley) Swart & Depurative hypertension (St; D; TB), leishmaniasis (Rs; DA; TB) \\
\hline \multirow[t]{3}{*}{ Cactaceae } & Epiphyllum phyllanthus (L.) Haw. & Body aches $(R ; I ; N B)$ \\
\hline & Pereskia bleo (HBK.) DC & Muscle aches (L; D; K) \\
\hline & Pereskia bleo (HBK.) DC & Stomachache (In; IK; K) \\
\hline Campanulaceae & $\begin{array}{l}\text { Centropogon coccineus (Hook.) Regel ex B.D. } \\
\text { Jacks. }\end{array}$ & Arthritis (St; D; TB) \\
\hline \multirow[t]{2}{*}{ Capparaceae } & Cleome serrata Jacq. & Topical warm bath (WP; D; K) \\
\hline & Cleome serrata Jacq. & Snakebite (L; IK; K) \\
\hline Caricaceae & Carica papaya L. & To improve blood circulation, to induce diuresis, to treat furuncles (F, L; IK; K) \\
\hline \multirow[t]{2}{*}{ Caryophyllaceae } & Drymaria cordata (L.) Willd. ex Schult. & Headache, stomachache (St, L; D; TB), fever (WP; D; TB) \\
\hline & Stellaria ovata Willd. ex Schltdl. & Worms (WP; D; TB) \\
\hline Chenopodiaceae & Chenopodium ambrosioides $\mathrm{L}$. & Stomachache (L; D; TB), to expel worms (L; Jc; TB) \\
\hline Clusiaceae & Symphonia globulifera L.f. & Bloody vomiting (St; I; NB), body pain (L; C; TB) \\
\hline Cochlospermaceae & Cochlospermum vitifolium (Willd.) Spr. & To improve blood circulation (Sd; l; K) \\
\hline \multirow[t]{5}{*}{ Commelinaceae } & Cochliostema odoratissimum Lem. & To regulate menstruation (L; D; NB) \\
\hline & Commelina diffusa Burm. f. & Common cold (WP; I; K) \\
\hline & Dichorisandra hexandra (Aubl.) Standl. & Snakebite (T; D; NB), internal and body pain (T; D; TB) \\
\hline & Tradescantia zanonia (L.) Sw. & Hemorrhage (S; DA; TB) \\
\hline & Tripogandra serrulata (Vahl) Handlos & Inflammation, fractures (WP; D; TB) \\
\hline \multirow[t]{2}{*}{ Convolvulaceae } & Ipomoea alba L. & Snakebite (L; C; TB), inflammation in snakebite (WP; D; TB) \\
\hline & Ipomoea indica (Burm. f.) Merr. & Laxative (L; D; NB) \\
\hline \multirow[t]{4}{*}{ Cucurbitaceae } & Cucurbita pepo L. & As bath, to accelerate children's growth (WP; I; K) \\
\hline & Gurania makoyana (Lem.) Cogn. & Leishmaniasis (St, L; D; TB), inflammation, liquid retention (L; D; TB) \\
\hline & Momordica charantia L. & Bile, to facilitate child birth, fever, hypertension (WP; D; TB) \\
\hline & Psiguria warscewiczii (Hook. f.) Wunderlin. & Shortness of breath (St; D; NB) \\
\hline Cyatheaceae & Cyathea petiolata (Hook.) Tryon & Ceremony to bathe sick children (WP; I; K) \\
\hline \multirow[t]{2}{*}{ Cycadaceae } & Zamia pseudoparasitica Yates & Emetic (T; D; K), muscle aches (St; DA; K) \\
\hline & Zamia sp. & Colic, constipation (St; D; NB) \\
\hline \multirow[t]{3}{*}{ Cyclanthaceae } & Carludovica palmata R. et P. & To prevent complications during childbirth (In; l; K) \\
\hline & Cyclanthus bipartitus Poit. & To avoid enlargement of uterus ( $F ; \mid K ; K)$ \\
\hline & Dicranopygium crinitum Harl. & To bathe sick children (WP; I; K) \\
\hline Cyperaceae & Cyperus luzulae (L.) Retz. & Eye infections, to avoid complications during childbirth (WP; I; K) \\
\hline \multirow[t]{2}{*}{ Dilleniaceae } & Davill kunthii St. Hil. & Colic in children $(\mathrm{Br}, \mathrm{F} ; \mathrm{l} ; \mathrm{K})$ \\
\hline & Tetracera volubilis L. & Body aches (St, L; D; TB) \\
\hline \multirow[t]{7}{*}{ Euphorbiaceae } & Acalypha hispida Burm. f. & To facilitate childbirth (F; IK; K) \\
\hline & Chamaesyce hirta (L.) Millsp. & Acne (L; ID; K) \\
\hline & Croton fragans HBK. & Common cold (L, F; ID; K) \\
\hline & Croton lobatus L. & Skin diseases (WP; I; K) \\
\hline & Jatropha sp. & Emetic (Sd; I; K) \\
\hline & Manhiot esculenta Crantz. & Acne (L; ID; K) \\
\hline & Phyllanthus urinaria $\mathrm{L}$. & Diarrhea (St; D; TB) \\
\hline \multirow[t]{10}{*}{ Fabaceae } & Bauhinia guianensis Aubl. & Diarrhea, bed wetting (St, L; D; TB) \\
\hline & Bauhinia reflexa Schery & Headache (St, L; D: TB) \\
\hline & Calliandra stipulacea Benth & Fever (WP; I; K) \\
\hline & Desmodium adscendens (Sw.) DC. & Conjunctivitis (WP; C; TB) \\
\hline & Desmodium axillare (Sw.) DC. & Postpartum aid to expel placenta (St; D; NB) \\
\hline & Machaerium sp. & Antitussive (WP; D; NB), aphthous ulcers of the mouth (S; NS; NB) \\
\hline & Mimosa pudica L. & Arthritis (St; I; NB) \\
\hline & Prioria copaifera Griseb. & Tonic, energizer $(\mathrm{F} ; \mathrm{I} ; \mathrm{K})$ \\
\hline & Senna fruticosa (Mill.) H.S. Irwin \& Barneby & Body aches (St, L; D; TB) \\
\hline & Senna reticulate (Willd.) Irwin \& Barneby. & Emetic (R; D; NB) \\
\hline \multirow[t]{2}{*}{ Flacourtiaceae } & Lindackeria laurina C. Presl. & Pneumonia (St; D; NB) \\
\hline & Xylosma sp. & Spider bites (St, R; I; NB) \\
\hline Gentianaceae & Schultesia lisianthoides (Griseb.) Benth. \& Hook & Pneumonia (St; I; NB) \\
\hline
\end{tabular}


Table 1 Medicinal plant inventory of Kuna, Ngöbe-Buglé, and Teribe Indians. (continued)

\begin{tabular}{|c|c|c|}
\hline Family & Specie & Application (plant part; preparation; Amerindian group) \\
\hline \multirow[t]{15}{*}{ Gesneriaceae } & Besleria laxiflora Benth. & Fever (L; D; TB) \\
\hline & Besleria solanoides Kunth & Fever (St; D; TB) \\
\hline & Chrysothemis friedrichsthaliana (Hanst.) H. E. & Muscle aches, inflammation of the joints (WP; I; K) \\
\hline & Chrysothemis pulcella (Donn ex Sims) Decne. & To facilitate childbirth (F; ; K) \\
\hline & Columnea nicaraguensis Oerst. & Chest pain (St; I; NB), fever, fatigue (St, L; D; TB) \\
\hline & Columnea sanguinolenta (Oerst.) Hanst. & Dysmenorrhea (St, L; D; TB) \\
\hline & $\begin{array}{l}\text { Columnea tulae Urb. var. tomentulosa (C. V. } \\
\text { Morton) B. D. Morley }\end{array}$ & Fever (St; D; TB), measles (St, L; D; TB), chicken pox (WP; Jc; TB) \\
\hline & Diastema scabrum (Poepp.) Benth. ex. Walp. & Fever, measles (St; D; TB), vertigo (WP; D; TB) \\
\hline & Drymonia macrophylla (Oerst.) H.E. Moore & Fever (St; D; TB) \\
\hline & Drymonia multiflora (Oerst. ex Hanst.) Wiehler & Inflammation, breast pain, painful breathing (WP; D; TB) \\
\hline & Drymonia serrulata (Jacq.) Mart. & Fever, headaches (St; D; NB), (St; Jc; TB) \\
\hline & Drymonia warscewicziana Hanst. & Difficulty in breathing (St; D; TB) \\
\hline & Gasteranthus acropodus (Donn. Sm.) Wiehler & Fever (WP; D; TB) \\
\hline & Gasteranthus imbricans (Donn. Sm.) Wiehler & Fever (St; D; TB) \\
\hline & Reldia veraguensis Wiehl. & Antiemetic (St; I; NB) \\
\hline \multirow[t]{2}{*}{ Gramineae } & Coix lacryma-jobi L. & Common cold (R, F; D; K) \\
\hline & Oplismenus burmanni (Retz.) Beauv. & Shortness of breath (WP; l; NB) \\
\hline Hamaedoraceae & Xyphidium caeruleum Aubl. & Antiemetic (St; I; NB), skin disorders (L; I; NB), to facilitate childbirth (St; I; TB) \\
\hline \multirow[t]{4}{*}{ Lamiaceae } & Hyptis sp. & Excipient (WP; NS; K) \\
\hline & Hyptis capitata Jacq. & Snakebite (L; IK; K) \\
\hline & Ocimum campechianum Mill. & Excipient (WP; NS; K) \\
\hline & Ocimum canum Sims. & Excipient, "enlargement of the mind" (L, F; ; K) \\
\hline Lecythidaceae & Gustavia superba (HBK.) Berg. & Mental disorders (F; IK; K) \\
\hline \multirow[t]{3}{*}{ Loganiaceae } & Spigelia anthelmia L. & Skin and eye infections (WP; D; K) \\
\hline & Spigelia humboldtiana Cham \& Schlecht. & Anthelmintic (WP; D; NB), pain and inflammation (St; D; TB), headache (WP; D; TB) \\
\hline & Strychnos sp. & Bee bites (St; D; NB) \\
\hline Lomariopsidaceae & Elaphoglossum herminieri (Bory ex F’ee) T. Moore & Burns (F; C; TB) \\
\hline Lythraceae & Cuphea epilobifolia Koehne & Rheumatism (St, L; D; TB) \\
\hline \multirow[t]{2}{*}{ Malpighiaceae } & Banisteriopsis muricata (Cav.) Cuatr. & Headache, fever (St; I; NB) \\
\hline & Heteropteris obovata (Small) Cuatr. et Croat. & Fever, headaches and diarrhea (St; D; NB) \\
\hline \multirow[t]{6}{*}{ Malvaceae } & Hibiscus rosa-sinensis $\mathrm{L}$. & To control size of uterus, "mental saturation" (F; l; K) \\
\hline & Hibiscus schizopetalus (Mast.) Hook. f. & To facilitate childbirth $(\mathrm{F} ; \mathrm{l} ; \mathrm{K})$ \\
\hline & Malvaviscus arboreus Cav. & Difficult childbirths (Br; D; NB) \\
\hline & Pavonia fruticosa (Mill.) Fawc. et Rendl. & Fever, common cold (WP; D; K) \\
\hline & Sida acuta Burm. f. var. acuta. & Tonic, antipyretic, alopecia in children (AP; D; K) \\
\hline & Sida rhombifolia L. & Inflammation, fractures (WP; D; TB), difficult birth (L; D; TB), to facilitate childbirth (St; D; TB) \\
\hline \multirow[t]{2}{*}{ Marantaceae } & Calathea lutea (Aubl.) G. Meyer & To increase learning capacity in children $(\mathrm{F} ; \mathrm{l} ; \mathrm{K})$ \\
\hline & Calathea warscewiczii (Mathieu) Koernicke. & Rubella (St; I; NB), snakebite (Rz, L; C; TB), painful wounds, inflammation (L; C; TB) \\
\hline \multirow[t]{2}{*}{ Marcgraviaceae } & Marcgravia nepenthoides Seemann. & Tiredness (Br; I; NB) \\
\hline & Souroubea sp. & Antidiarrhoeic (St; I; NB) \\
\hline \multirow[t]{5}{*}{ Melastomataceae } & Arthrostemma ciliatum Ruiz \& Pav. & Urinary infections (St, L; D; TB) \\
\hline & Bellucia pentamera Naudin & Chicken pox, measles (St; D; TB) \\
\hline & Blakea foliacea Gleason. & Diarrhea (St; D; NB) \\
\hline & Ossaea quinquenervia (Mill.) Cogn. & Fever (St; D; TB) \\
\hline & Triolena hirsuta (Benth.) Triana & Nose bleeding (St; D; TB) \\
\hline \multirow[t]{3}{*}{ Meliaceae } & Carapa guianensis Aubl. & Furuncles, skin infections (S; C; TB) \\
\hline & Cedrela odorata $\mathrm{L}$. & Leishmaniasis (St; D; TB) \\
\hline & Guarea multiflora A. Juss. & Hepatic disorders, emetic (St; I; NB) \\
\hline \multirow[t]{2}{*}{ Menispermaceae } & Cissampelos pareira $\mathrm{L}$. & As a drink when feeling sick on waking up (St; D; NB) \\
\hline & Cissampelos tropaeofolia DC. & Snakebite (St; D; NB), diarrhea (St; D; TB) \\
\hline Monimiaceae & Siparuna sp. & Fever $(\mathrm{L} ; \mathrm{I} ; \mathrm{K})$ \\
\hline \multirow{3}{*}{ Moraceae } & Cecropia peltata $\mathrm{L}$. & Headache, "mental saturation” (In; D; K) \\
\hline & Dorstenia contrajerva L. & Snakebite, muscle aches (WP; D; K) \\
\hline & Ficus insipid Willd. & Skin diseases (St; I; K) \\
\hline \multirow[t]{5}{*}{ Musaceae } & Heliconia hirsuta L. F. & Antidiarrhoeic (R; I; NB) \\
\hline & Heliconia mariae Hook. f. & To improve blood circulation (F; D; K) \\
\hline & Heliconia platystachys Baker & To facilitate childbirth (F; l; K) \\
\hline & Heliconia vaginalis Benth. & Drunk when fetus is found to be incorrectly positioned (WP; D; K) \\
\hline & Musa sapientum L. & Urinary infection (Ht; D; TB) \\
\hline Myrtaceae & Psidium guajava L. & Diarrhea (Br; D; TB) \\
\hline Olaceae & Heisteria macrophylla Oerst. & Abdominal cramps (St; I; NB) \\
\hline Orchidaceae & Dichaea muricata (SW.) Lindl. & Anthelmintic (WP; I; NB) \\
\hline
\end{tabular}


Table 1 Medicinal plant inventory of Kuna, Ngöbe-Buglé, and Teribe Indians. (continued)

\begin{tabular}{|c|c|c|}
\hline Family & Specie & Application (plant part; preparation; Amerindian group) \\
\hline & Epidendrum difforme Jacq. & Rubella (L; I; NB) \\
\hline & Maxillaria sp. & Fatigue, exhaustion (L; I; NB) \\
\hline & Octomeria sp. & Antiemetic (NS; I; NB) \\
\hline \multirow[t]{2}{*}{ Palmae } & Bactris sp. & Difficulties in breathing (St; D; NB) \\
\hline & Cocos nucifera $\mathrm{L}$. & Intense back pain (R; D; TB) \\
\hline \multirow[t]{5}{*}{ Passifloraceae } & Passiflora costaricencis Killip & Chest pain (L; D; TB), body aches (St; D; TB); pain, snakebite (St, L; D; TB) \\
\hline & Passiflora pediculata Mast. & Snakebite (St; D; NB) \\
\hline & Passiflora quadrangularis L. & Anti-inflammatory (St; D; NB) \\
\hline & Passiflora sexflora Juss. & Snakebite (WP; D; NB) \\
\hline & Passiflora vitifolia HBK. & “Mental saturation” (F; I; K), snakebite (St; l; NB) \\
\hline \multirow[t]{2}{*}{ Phytolaccaceae } & Rivina humilis L. & Cold (St, L; D; TB) \\
\hline & Petiveria alliacea $\mathrm{L}$. & Ritual ceremonies (St, L; D; NB) \\
\hline \multirow[t]{9}{*}{ Piperaceae } & Peperomia aff. ebingeri Yunck. & Measles (WP; l; TB) \\
\hline & Peperomia pellucida (L.) Kunth & Foot inflammation (WP; D; TB) \\
\hline & Peperomia rotundifolia (L.) H. B. K. & Rash (L; D; NB) \\
\hline & Piper auritum HBK. & Common cold (In; I; K) \\
\hline & Piper erubescentispicum Trel. & Headache (L; D; TB) \\
\hline & Piper hispidum Sw. & Eyes infection, muscle aches (L; D; K), fever (St; D; TB), to expel worms (St; C; TB) \\
\hline & Piper multiplinervium C. DC. & Body- and stomachaches $(\mathrm{L} ; \mathrm{l} ; \mathrm{K})$ \\
\hline & Piper peltatum $\mathrm{L}$. & Infected wound (L; D; TB) \\
\hline & Piper tuberculatum Jacq. & Liver pains (In, L; IK, D; K) \\
\hline \multirow[t]{3}{*}{ Polygalaceae } & Polygala panamensis Chodat. & Sore eyes (WP; D; NB) \\
\hline & Polygala paniculata $\mathrm{L}$. & Fever (WP; D; TB) \\
\hline & Securidaca diversifolia (L.) S. F. Blake & Toothache (L; D; TB) \\
\hline \multirow[t]{2}{*}{ Polypodiaceae } & Niphidium crassifolium (L.) Lellinger & Fever (L; D; NB) \\
\hline & Polypodium sp. & Difficulties in breathing (T; NS; NB) \\
\hline Rhizophoraceae & Rhizophora mangle L. & Healing of bone fracture ( $R ; \mathrm{I} ; \mathrm{K})$ \\
\hline \multirow[t]{17}{*}{ Rubiaceae } & Borreria laevis (Lam.) Griseb. & Muscle and bone aches, inflammation, skin diseases, acne (WP; D; K) \\
\hline & Cephaelis elata Sw. & Dizziness, hallucination, dementia (St; D; NB) \\
\hline & Cephaelis tomentosa (Aubl.) Vahl. & Dizziness, hallucination, rubella (St, R; D; NB) \\
\hline & Chione panamensis Steyermark. & Ritual ceremonies (R; I; NB) \\
\hline & Coffea arabica L. & Fever (L; l; K) \\
\hline & Faramea eurycarpa Don. Sm. & Pruritous (St; D; NB) \\
\hline & Genipa americana $\mathrm{L}$. & Drunk by mother to regulate growth of fetus $(\mathrm{L} ; \mathrm{l} ; \mathrm{K})$, to treat weakness in girls (F; NS; K) \\
\hline & Hamelia patens var. glabra. Oerst. & Snakebite, postpartum aid to relieve pain (St; I; NB) \\
\hline & Hoffmannia vesiculifera Standl. & Headache, body aches (St, R; D; NB), to induce and facilitate childbirth (St; D; TB) \\
\hline & Manettia reclinata L. Mant. & Renal analgesic (St; D; NB), fever (St; D; TB) \\
\hline & $\begin{array}{l}\text { Notopleura anomothyrsa (K. Schum. \& Donn. } \\
\text { Sm.) C. M. Taylor }\end{array}$ & Fever (St; D; TB), stomachache (St, L; D; TB) \\
\hline & Palicourea guianensis Aubl. & Mental disorders $(\mathrm{F} ; \mathrm{l} ; \mathrm{K})$ \\
\hline & Pentagonia pinnatifida Seem. & Fever $(\mathrm{R} ; \mathrm{I} ; \mathrm{K})$, to facilitate childbirth and menstruation $(\mathrm{F} ; \mathrm{IK} ; \mathrm{K})$ \\
\hline & Psychotria emetica L.f. & Chicken pox, fever (R; D; TB) \\
\hline & Psychotria psychotriifolia (Seem.) Standl. & Fever (St; D; TB) \\
\hline & Psychotria uliginosa Sw. & Stomachache, fever (L; I; NB) \\
\hline & Sabicea villosa R. et S. & Rheumatism (St; I; NB) \\
\hline Rutaceae & Citrus limon (L.) Burm. f. & Common cold, cough, breathing difficulties (P; NS; K) \\
\hline \multirow[t]{2}{*}{ Sapindaceae } & Sapindus saponaria $\mathrm{L}$. & Skin diseases, colds (F; I; K) \\
\hline & Serjania rhombea Radlk. & Dizziness (St; I; NB) \\
\hline \multirow[t]{3}{*}{ Scrophulariaceae } & Alectra aspera (Cham. et Schlecht.) L. O. Williams. & Difficulties in breathing (St; l; NB) \\
\hline & Scoparia dulcis L. & Fever (St; D; TB), chicken pox (L; D; TB) \\
\hline & Russelia sarmentosa Jacq. & Snakebite (WP; D; NB) \\
\hline Selaginellaceae & Selaginella sp. & Fever, weakness, muscle aches and eye infection (L; IK; K) \\
\hline \multirow[t]{2}{*}{ Simaroubaceae } & Picramnia allenii D. M. Porter. & Shortness of breath (St; D; NB) \\
\hline & Simaba cedron Planch. & Fever, malaria, snakebites (F; I; NB) \\
\hline Smilacaceae & Smilax chiriquensis C. V. Morton & Weakness, malnutrition (Rz; D; TB) \\
\hline \multirow[t]{5}{*}{ Solanaceae } & $\begin{array}{l}\text { Capsicum annuum L. var. aviculare (Dierb.) } \\
\text { D'Arcy et Eshb. }\end{array}$ & Serious ailments (F, L; IK, ID; K) \\
\hline & Cestrum nocturnum $\mathrm{L}$. & Fever (L; D; TB) \\
\hline & Cuatresia exiguiflora (D’Arcy) Hunz. & Postpartum birth (St; D; TB) \\
\hline & Cyphomandra hartwegii (Miers) Dun. & Snakebite (St; I; NB) \\
\hline & $\begin{array}{l}\text { Lycianthes amatitlanensis (J.M. Coult. \& Donn. } \\
\text { Sm.) Bitter }\end{array}$ & Headache (St, L; NS; TB) \\
\hline
\end{tabular}


Table 1 Medicinal plant inventory of Kuna, Ngöbe-Buglé, and Teribe Indians. (continued)

\begin{tabular}{|c|c|c|}
\hline \multirow[t]{4}{*}{ Family } & Specie & Application (plant part; preparation; Amerindian group) \\
\hline & Solanum lancaeifolium Jacq. & Muscle and stomachaches (WP; D; K); shortness of breath (R; D; NB) \\
\hline & Witheringia correana D’Arcy & Fever (St; D; TB) \\
\hline & Witheringia solanacea L'Her. & Anthelmintic (WP; l; NB) \\
\hline \multirow[t]{2}{*}{ Sterculiaceae } & Theobroma cacao L. & To facilitate childbirth (St; IK; K), bleeding (F; C; TB) \\
\hline & Herrania purpurea (Pittier) R. E. Schultes & To facilitate childbirth (AP; IK; K) \\
\hline Tectariaceae & Cyclopeltis semicordata (Sw.) J. Sm. & Burns (Sc; DA; TB) \\
\hline \multirow[t]{2}{*}{ Theophrastaceae } & Clavija costaricana Pittier & To expel worms (St; D; TB) \\
\hline & Clavzja sp. & Women's ailment (L; I; NB) \\
\hline Tiliaceae & Heliocarpus americanus L. & To facilitate child birth (St; I; TB) \\
\hline \multirow[t]{4}{*}{ Urticaceae } & Pilea imparifolia Wedd. & Diarrhea (St; D; NB) \\
\hline & Pilea microphylla (L.) Liebm. & Chicken pox (St, L; D; TB), warts (WP; C; TB), measles (WP; D; TB) \\
\hline & Urera caracasana (Jacq.) Griseb & Foot pain (L; DA; TB) \\
\hline & Urera laciniata (Goud.) Wedd. & Body aches (L; NS; K) \\
\hline \multirow[t]{3}{*}{ Verbenaceae } & Lantana hispida Kunth & Placenta retention $(\mathrm{Fl} ; \mathrm{I} ; \mathrm{K})$ \\
\hline & Lantana trifolia L. & Fever (St, L; D; TB) \\
\hline & Stachytarpheta jamaicensis (L.) Vahl & Neck pain, muscle pain (L; D; TB) \\
\hline Vittariaceae & Vittaria lineate (L.) Sw. & Headaches (L; D; NB) \\
\hline Zamiaceae & Zamia skinneri Warsz. ex A. Dietr. & Wound healing (Rz; D; TB) \\
\hline \multirow[t]{4}{*}{ Zingiberaceae } & Costus ruber Griseb & To calm pain after childbirth (St; I; NB) \\
\hline & Costus villosissimus Jacq. & To calm pain after childbirth (St; I; NB) \\
\hline & Dimerocostus strobilaceus Kuntze & To improve blood circulation (FI, L; I; K) \\
\hline & Zingiber officinale Roscoe & Pneumonia, toothache (Rz; D; TB) \\
\hline
\end{tabular}

FI: flower; L: leave; AP: aerial parts; St: stem; WP: whole plant; F: fruit; P: peel; R: root; Rz: rhizomes; In: Inflorescence; Pt: petioles; Br: branch; S: sap; Sd: seed; T: tuber; Sc: scales; VI; vines; Ht: heart; I: infusion; D: decoction; C: cataplasm; IK: Ina kuamakalet; ID: Ina dibialet; Jc: juice; Rs: resin; DA: direct application; NS: not specified; K: Kuna Indians; NB: NgäbeBuglé; TB: Teribes [14-17]

ceae, Rubiaceae, Fabaceae, Piperaceae, and Simaroubaceae, which are some of the major plant families in Panama. A total of 160 new compounds belonging to a wide variety of chemical structures: acetogenins, flavonoids, alkaloids, coumarins, terpenoids, quinones, benzophenones, iridoids, and quassinoids were isolated from Panamanian plants. Noteworthy are the new compounds isolated from plants such as Marila pluricostata Standl. \& L. O. Williams, Tovomita longifolia (Rich.) Hochr., Vismia macrophylla Kunth., Bonamia trichantha Hallier f., Sloanea zuliaensis Pittier, Adenaria floribunda Kunth., Triplaris cumingiana Fisch. \& C.A. Mey. ex Mey., which exhibited cytotoxicity to specific cancer cell lines; and from Monnina sylvatica Schltdl. \& Cham, Piper dilatatum Rich., Lonchocarpus chiricanus Pittier, Erythrina berteroana Urb., and Cordia linnaei Stearn, which exhibited antifungal activity. Additionally, new compounds isolated from Siparuna thecaphora (Poepp. \& Endl.) A. DC., Calycolpus warzewiczianus O. Berg, Clidemia sericea D. Don, Cornutia grandifolia var. intermedia, Arrabidaea patellifera (Schltdl.) Sandwith were identified as antiplasmodials; and chemical evaluation of Guatteria dumentorum R.E. Fr. revealed new compounds toxic to Leishmania mexicana. Moreover, novel dimeric secoiridoid lisianthioside from Lisianthus jefensis with antimicrobial activity, larvicidal purpureacin from Annona purpurea against Aedes aegypti, antifungal xanthones from Marila laxiflora, antifungal and larvicidal naphthoquinones and naphthoxirene from Cordia linnaei, and antifungal benzoic acid derivatives from Piper dilatum are also worth mentioning. Thus, these data show that extensive pharmacognostic research on Panamanian flora can increase the probabilities of discovering new lead compounds of therapeutic importance.

\section{Conclusions}

$\nabla$

From the results presented, it is immediately obvious that the Panamanian flora is still an untapped valuable source of new bioactive natural products. Most of the work reported here has been the result of many collaborative efforts with scientists worldwide. During the last quarter century, a total of approximately 390 compounds from 86 plants have been isolated to date, of which 160 are new to the literature. These belong to very diverse compound classes: naphthoquinones, flavonoids, biphenyls, coumarins, acetogenins, xanthones, benzoic acid derivatives, monoterpenes, triterpenes, saponins, alkaloids, amides, procyanidins, quinones, amides, iridoids, tannins, dimeric secoiridoids, and quassinoids. Among the isolated compounds, 155 exhibited a range of biological activities. Ethnopharmacological information gathered and extensive screening of Panamanian flora have indicated that many species have interesting activities and that much remains to be done. The potential of discovering new lead compounds is clearly evident.

The richness of plant biodiversity, high percentage of endemic species and a good legal framework for accessing genetic resources make Panama a unique place for international collaboration. For example a European Union project within the Framework of Seventh Program called AGROCOS is currently being executed in Panama in collaboration with a consortium of industries and academic institutions in Europe, South Africa, and Panama with the aim to discover novel compounds for the agrochemical and cosmetic industries.

\section{Supporting Information}

Table 1S provides a summary of novel compounds from Panamanian flora, but not tested for biological activity. 
Table 2 Chemistry and biological activity of selected Panamanian plants.

\begin{tabular}{|c|c|c|c|c|c|}
\hline Family & Species & New compounds & Known compounds & Biological activity & Ref. \\
\hline Amaryllidaceae & $\begin{array}{l}\text { Hymenocallis littoralis } \\
\text { (Jacq.) Salisb. = Pancrati- } \\
\text { um littorale Jacq. }\end{array}$ & & 7,4'-dihydroxy-8-methylflavan (1) & DPPH: (1) & {$[32]$} \\
\hline Anacardiaceae & $\begin{array}{l}\text { Mosquitoxylon jamai- } \\
\text { cense Krug \& Urb. }\end{array}$ & & $\begin{array}{l}\text { phloridzin (2), 4-hydroxy benzene- } \\
\text { propanal (3), trilobatin (4), querce- } \\
\text { tine-3-O- } \beta \text {-D-galactoside (5) }\end{array}$ & AP: $P f(2)$ & [33] \\
\hline \multirow[t]{4}{*}{ Annonaceae } & $\begin{array}{l}\text { Annona purpurea Moc. \& } \\
\text { Sessé ex Dunal }\end{array}$ & $\begin{array}{l}\text { purpureacin } 1(6) \\
\text { purpureacin } 2(7)\end{array}$ & $\begin{array}{l}\text { rolliniastatin } 1(\mathbf{8}) \text {, bullatacin }(\mathbf{9}) \text {, } \\
\text { cherimolin }(\mathbf{1 0}) \text {, sylvaticin }(\mathbf{1 1})\end{array}$ & $\begin{array}{l}\text { AB: } B s(6) \\
\text { AF: } C a(6,7,9-11)\end{array}$ & [34] \\
\hline & $\begin{array}{l}\text { Duguetia panamensis } \\
\text { Standl. }\end{array}$ & & 2,4,5-trimethoxystyrene (12) & TBS: (12) & [35] \\
\hline & $\begin{array}{l}\text { Guatteria amplifolia } \\
\text { Triana and Planch }\end{array}$ & & $\begin{array}{l}\text { xylopine (13), nornuciferine (14), } \\
\text { lysicamine (15), laudanoscine (16) }\end{array}$ & L: $\operatorname{Lm}, \operatorname{Lp}(13,14)$ & [36] \\
\hline & $\begin{array}{l}\text { Guatteria dumentorum } \\
\text { R.E. Fr. }\end{array}$ & $\begin{array}{l}\text { (+)-isodomesticine (17), } \\
(+) \text {-norisodomesticine } \\
(\mathbf{1 8}),(+)-\text {-nantenine (19), } \\
(+) \text {-neolitsine (20), (+)-lirio- } \\
\text { ferine (21), (+)-N-methyl- } \\
\text { laurotetanine (22), (+)-nor- } \\
\text { lirioferine (23), (+)-isobol- } \\
\text { dine (24), (+)-reticuline } \\
\text { (25) }\end{array}$ & $\begin{array}{l}\text { cryptodorine (26), nornantenine } \\
\text { (27) }\end{array}$ & $\begin{array}{l}\text { L: } \operatorname{Lm}, \operatorname{Lp}(\mathbf{2 6}, \mathbf{2 7}) \\
\operatorname{Lm}(\mathbf{1 7 - 2 0})\end{array}$ & {$[36,37]$} \\
\hline Apocynaceae & $\begin{array}{l}\text { Stemmadenia } \\
\text { robinsonii } \\
\text { Woodson }\end{array}$ & 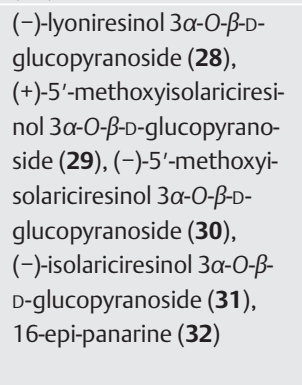 & 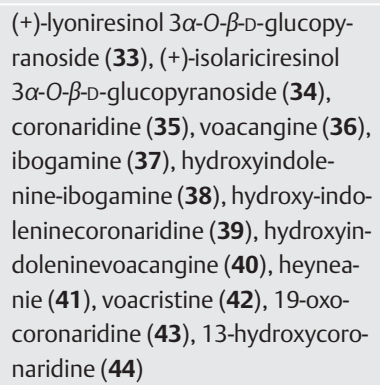 & $\begin{array}{l}\text { AB: } B s(42,44) \\
\text { TBS: }(36)\end{array}$ & {$[38,39]$} \\
\hline Asteraceae & $\begin{array}{l}\text { Baccharis pedunculata } \\
\text { (Mill.) Cabrera }\end{array}$ & & $\begin{array}{l}\text { lachnophyllum lactone (45), } \\
\text { ulopterol 3-methylether flavones } \\
\text { (46), ulopterol (47) }\end{array}$ & $\begin{array}{l}\text { AF: } C c(46,47), C a(45, \\
\text { 46), Ef }(45,47), M g(45) \\
\text { Toxic to human } \\
\text { keratinocytes: (45) }\end{array}$ & [40] \\
\hline \multirow[t]{2}{*}{ Bignoniaceae } & $\begin{array}{l}\text { Arrabidaea patellifera } \\
\text { (Schltdl.) Sandwith }\end{array}$ & $\begin{array}{l}\text { (3'-O-p-hydroxybenzoyl- } \\
\text { mangiferin (48), 3'-O- } \\
\text { trans-coumaroylmangifer- } \\
\text { in (49), 6'-O-trans-coumar- } \\
\text { oylmangiferin (50), 3'-O- } \\
\text { trans-cinnamoylmangiferin } \\
\text { (51), 3'-O-trans-caffeoyl- } \\
\text { mangiferin (52), 3'-O-ben- } \\
\text { zoylmangiferin (53) }\end{array}$ & mangiferin (54), isomangiferin (55) & $\begin{array}{l}\text { AP: } P f(48-54) ; D P P H, \\
\text { ALP: }(48-54)\end{array}$ & [41] \\
\hline & Jacaranda caucana Pittier & $\begin{array}{l}\text { 6'-O-(cis-1,4-dihydroxycy- } \\
\text { clohexanacetyl) acteoside } \\
\text { (56), 6'-O-(1-hydroxy-4- } \\
\text { oxo-cyclohexanacetyl) } \\
\text { acteoside (57), 4-O-rham- } \\
\text { nosyl-7S, 8R-7,8'-erythro- } \\
\text { sisymbrifolin (58) }\end{array}$ & $\begin{array}{l}\text { protocatechuic acid (59), acteoside } \\
\text { (60), jionoside D (61), isoacteoside } \\
\text { (62), martynoside (63) }\end{array}$ & DPPH, ALP: (56-63) & {$[42]$} \\
\hline \multirow[t]{3}{*}{ Boraginaceae } & $\begin{array}{l}\text { Cordia alliodora Ruiz \& } \\
\text { Pavon }\end{array}$ & $\begin{array}{l}\text { 1-(3'-methoxypropanoyl)- } \\
\text { 2,4,5-trimethoxybenzene } \\
\text { (64), 2-(2Z)-(3-hydroxy-3,7- } \\
\text { dimethylocta-2, 6-dienyl)- } \\
\text { 1,4-benzenediol (65) }\end{array}$ & & $\begin{array}{l}\text { AF: } C c(64,65) \\
\text { LC: } A a(64)\end{array}$ & [43] \\
\hline & Cordia linnaei Stearn & $\begin{array}{l}\text { cordiaquinona } \mathrm{E}(66) \text {, } \\
\text { cordiaquinona } \mathrm{F}(67) \text {, } \\
\text { cordiaquinona } \mathrm{G}(68) \text {, } \\
\text { cordiaquinona } \mathrm{H}(\mathbf{6 9})\end{array}$ & $\begin{array}{l}\text { cordiaquinona } B(70) \text {, } \\
\text { naftoxireno (71) }\end{array}$ & AF: $C c, C a(66-70)$ & [44] \\
\hline & Cordia spinescens L. & & $\begin{array}{l}\text { magnesium lithospermate (72), } \\
\text { calcium rosmarinate (73), } \\
\text { magnesium rosmarinate (74) }\end{array}$ & HIV-1 RT: (72-74) & {$[45,46]$} \\
\hline
\end{tabular}


Table 2 Chemistry and biological activity of selected Panamanian plants. (continued)

\begin{tabular}{|c|c|c|c|c|c|}
\hline Family & Species & New compounds & Known compounds & Biological activity & Ref \\
\hline \multirow[t]{2}{*}{ Calophyllaceae } & Marila laxiflora Rusby & & $\begin{array}{l}\text { 5-hydroxy-1-methoxyxanthone } \\
\text { (75), 1,5-dihydroxyxanthone (76), } \\
\text { 1,6-dihydroxy-5-methoxyxanthone } \\
\text { (77), betulinic acid (78), rhamnetin } \\
\text { (79), 2-(3,3-dimethylallyl)-1,3,5,6- } \\
\text { tetrahydroxyxanthone (80), 3,4-di- } \\
\text { hydroxy-benzoic acid (81) }\end{array}$ & AF: $C c(75-77)$ & {$[47]$} \\
\hline & $\begin{array}{l}\text { Marila pluricostata } \\
\text { Standl. \& L. O. } \\
\text { Williams }\end{array}$ & $\begin{array}{l}\text { 5-hydroxy-8,8-dimethyl-4- } \\
\text { phenyl-9,10-dihydro-8H- } \\
\text { pyrano-[2,3-f]chromen-2- } \\
\text { one (82), 5-hydroxy-8,8-di- } \\
\text { methyl-4-phenyl-6-propio- } \\
\text { nyl-9,10-dihydro-8H-pyra- } \\
\text { no-[2,3-f]chromen-2-one } \\
\text { (83), and 5,7-dihydroxy-8- } \\
\text { (3-methylbut-2-enyl)-4- } \\
\text { phenylchromen-2-one } \\
\text { (84), pluricostatic acid (85) }\end{array}$ & $\begin{array}{l}\text { mammeisin (86), isomammeisin } \\
\text { (87), mammeigin (88), MAB 5 (89), } \\
\text { mesuagin (90), isomesuol (91), } \\
\text { MAB } 1 \text { (92), mesuol (93), mammea } \\
\text { A/BB (94), isodispar B (95), cyclo- } \\
\text { mammeisin (96), disparinol A (97), } \\
\text { MAB } 3 \text { (98), and mesuol cyclo F } \\
\text { (99), squalene (100), friedelin } \\
\text { (101), 4-epi-friedelin (102), cano- } \\
\text { phyllal (103), friedelinol (104), } \\
\text { canophyllol (105), 3-oxo-friedelan- } \\
\text { 28 oic acid (106), D:A-friedo-3,4- } \\
\text { seco-olean-3-oic acid (107), epi-be- } \\
\text { tulinic acid (108), betulinic acid } \\
\text { (109), } \beta \text {-sistosterol (110), stigmas- } \\
\text { terol (111), 3-O- } \beta \text {-glucopyranosyl- } \\
\text { sistosterol (112) }\end{array}$ & $\begin{array}{l}\text { AC: MCF-7, H-460, } \\
\text { SF-268 (82-87, 91-112) }\end{array}$ & {$[48,49]$} \\
\hline \multirow[t]{3}{*}{ Clusiaceae } & $\begin{array}{l}\text { Tovomita longifolia } \\
\text { (Rich.) Hochr. }\end{array}$ & $\begin{array}{l}\text { (E)-3-(2-hydroxy-7-methyl- } \\
\text { 3-methyleneoct-6-enyl)- } \\
\text { 2,4,6-trihydroxybenzophe- } \\
\text { none (113), (E)-3-(6-hy- } \\
\text { droxy-3,7-dimethylocta- } \\
\text { 2,7-dienyl)-2,4,6-trihy- } \\
\text { droxybenzophenone } \\
\text { (114), 8-benzoyl-2-(4- } \\
\text { methylpenten-3-yl)chro- } \\
\text { mane-3,5,7-triol (115), } \\
\text { and 5-benzoyl-1,1,4a-tri- } \\
\text { methyl-2,3,4,4a,9,9a-hexa- } \\
\text { hydro-1H-xanthene-6,8- } \\
\text { diol (116) }\end{array}$ & $\begin{array}{l}\text { 4-geranyloxy-2,6-dihydroxybenzo- } \\
\text { phenone (117) and 3-geranyl-2,4,6- } \\
\text { trihydroxybenzophenone (118) }\end{array}$ & $\begin{array}{l}\text { AC: MCF-7, H-460, } \\
\text { SF-268 }(\mathbf{1 1 3}, \mathbf{1 1 5}, \mathbf{1 1 7}) \\
\text { AB: Ms }(\mathbf{1 1 5}-\mathbf{1 1 8}), \\
\text { Kp }(\mathbf{1 1 5}, \mathbf{1 1 6}) \\
\text { Sg }(\mathbf{1 1 6}), P a(\mathbf{1 1 6}), \\
\text { Sa (117) }\end{array}$ & {$[50]$} \\
\hline & $\begin{array}{l}\text { Vismia macrophylla } \\
\text { Kunth }\end{array}$ & ferruginin C (119) & $\begin{array}{l}\text { ferruginins } A(120) \text { and } B(121) \text {, } \\
\text { vismin (122), harunganin (123) }\end{array}$ & $\begin{array}{l}\text { AC: MCF-7, H-460, } \\
\text { SF-268 (119-123) }\end{array}$ & [51] \\
\hline & $\begin{array}{l}\text { Vismia baccifera (L.) } \\
\text { Triana \& Planch. }\end{array}$ & & $\begin{array}{l}\text { vismione } B(124) \text {, deacetylvismione } \\
A(125) \text { and } H(126) \text {, bivismiaqui- } \\
\text { none (127), vismiaquinone (128) }\end{array}$ & $\begin{array}{l}\text { AC: MCF-7, H-460, } \\
\text { SF-268 (124-126) }\end{array}$ & {$[51]$} \\
\hline Convolvulaceae & $\begin{array}{l}\text { Bonamia trichantha } \\
\text { Hallier } \mathrm{f} \text {. }\end{array}$ & $\begin{array}{l}\text { trichanthins A (129), B } \\
\text { (130), C (131), and D (132) }\end{array}$ & & $\begin{array}{l}\text { AC: MCF-7, H-460, } \\
\text { SF-268 }(129,130,132)\end{array}$ & {$[52]$} \\
\hline Elaeocarpaceae & Sloanea zuliaensis Pittier & 2-deoxycucurbitacin-D (133) & $\begin{array}{l}\text { cucurbitacin D (134), 25-acetyl- } \\
\text { cucurbitacin F (135) }\end{array}$ & $\begin{array}{l}\text { AC: MCF-7, H-460, } \\
\text { SF-268 (133-135) }\end{array}$ & [53] \\
\hline Euphorbiaceae & $\begin{array}{l}\text { Chamaesyce hyssopifolia } \\
\text { (L.) Small }\end{array}$ & & $\begin{array}{l}\text { quercetin (136), quercetin 3-O- } \alpha \text {-L- } \\
\text { arabinopyranoside (137), quercetin } \\
\text { 3-O- } \beta \text {-D-xylopyranoside (138), quer- } \\
\text { cetin 3-O- } \beta \text {-D-glucopyranoside } \\
\text { (139), quercetin 3-O- } \beta \text {-D-galacto- } \\
\text { pyranoside (140), apigenin 7-O- } \beta \text {-D- } \\
\text { glucopyranoside (141), kaempferol } \\
\text { 3-O- } \beta \text {-D-glucopyranoside (142), gal- } \\
\text { lic acid (143), gallic acid methyl es- } \\
\text { ter (144), corilagin (145), 1,3,4,6- } \\
\text { tetra-O-galloyl- } \beta \text {-D-glucopyranose } \\
\text { (146) }\end{array}$ & HIV-1 RT: (136-138) & {$[45,46]$} \\
\hline Fabaceae & $\begin{array}{l}\text { Andira inermis (W. } \\
\text { Wright) Kunth. ex DC. }\end{array}$ & & $\begin{array}{l}\text { formononetin (147), calicosin } \\
\text { (148), prunetin (149), biocanin } \\
\text { (150), genistein (151), pratensein } \\
\text { (152) }\end{array}$ & AP: $P f(148,151)$ & {$[54]$} \\
\hline
\end{tabular}


Table 2 Chemistry and biological activity of selected Panamanian plants. (continued)

\begin{tabular}{|c|c|c|c|c|c|}
\hline \multirow[t]{5}{*}{ Family } & Species & New compounds & Known compounds & Biological activity & Ref.] \\
\hline & Erythrina berteroana Urb. & $\begin{array}{l}\text { 5,7-dihydroxy-3-[5-hydroxy-4- } \\
\text { methoxy-3-(3-methyl-2-bute- } \\
\text { nyl)phenyl]-2,3-dihydro-4H-1- } \\
\text { benzopyran-4-one (153), sig- } \\
\text { moidin B (154) }\end{array}$ & & AF: $C c(153,154)$ & {$[55,56]$} \\
\hline & $\begin{array}{l}\text { Lonchocarpus chiricanus } \\
\text { Pittier }\end{array}$ & $\begin{array}{l}\text { chiricanines A-E (155- } \\
\text { 159) }\end{array}$ & $\begin{array}{l}\text { longistylines C (160) and D (161), } \\
\text { 3,5-dimethoxystilbene (162) }\end{array}$ & $\begin{array}{l}\text { AF: } C c(155), \text { LC: } A a \\
(162)\end{array}$ & [57] \\
\hline & $\begin{array}{l}\text { Myrospermum frutescens } \\
\text { Jacq. }\end{array}$ & 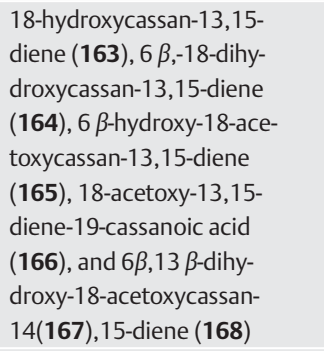 & & AT: $T c(163-168)$ & [58] \\
\hline & $\begin{array}{l}\text { Swartzia simplex (Sw.) } \\
\text { Spreng. }\end{array}$ & $\begin{array}{l}\beta \text {-D-glucopyranosyl[ } \beta \text {-D- } \\
\text { glucuronopyranosyl } \\
(1 \rightarrow 3)] \text {-3- } \beta \text {-hydroxyolean- } \\
\text { 12-en-23-al-28-oate }(169) \text {, } \\
\beta \text {-D-glucopyranosyl[ } \beta \text {-D-xy- } \\
\text { lopyranosyl-( }(1 \rightarrow 2)(\alpha \text {-L- } \\
\text { rhamnopyranosyl }(1 \rightarrow 3) \text { - } \\
\beta \text {-D-glucuropyranosyl } \\
(1 \rightarrow 3)] \text {-3 } \beta \text {-hydroxyolean- } \\
12 \text {-en-28-oate }(170), \beta \text {-D- } \\
\text { glucopyranosyl[ } \beta \text {-D-glucur- } \\
\text { onopyranosyl }(1 \rightarrow 4)] \text {-[ } \beta \text {-D- } \\
\text { glucopyranosyl-( }(1 \rightarrow 3)]- \\
\text { 3 } \beta \text {-hydroxyolean-12-en-28- } \\
\text { oate }(171)\end{array}$ & $\begin{array}{l}\text { [ } \alpha \text {--- rhamnopyranosyl-( }(1 \rightarrow 3) \text { - } \beta \text {-D- } \\
\text { glucopyranosyl- }(1 \rightarrow 3)]-3 \beta \text {-hy- } \\
\text { droxyolean-12-en-28-oic acid }(\mathbf{1 7 2}) \text {; } \\
\beta \text {-D-glucopyranosyl[ } \beta \text {-D-glucurono- } \\
\text { pyranosyl }(1 \rightarrow 3) \text {-3- } \beta \text {-hydroxyo- } \\
\text { lean-12-en-28-oate }(\mathbf{1 7 3}), \beta \text {-D-glu- } \\
\text { copyranosyl [ } \alpha \text {--L-rhamnopyranosyl } \\
(1 \rightarrow 3) \text { - } \beta \text {-D-glucuropyranosyl } \\
(1 \rightarrow 3)]-3 \beta \text {-hydroxyolean-12-en-28- } \\
\text { oate }(\mathbf{1 7 4}), \beta \text {-D-glucopyranosyl } \\
{[\beta \text {-D-glucopyranosyl }(1 \rightarrow 3)]} \\
3 \beta \text {-hydroxyolean-12-en-23-oic-28- } \\
\text { oate }(\mathbf{1 7 5})\end{array}$ & MC: $B g(171,172)$ & [59] \\
\hline Gentianaceae & $\begin{array}{l}\text { Lisianthius seemannii } \\
\text { (Griseb) O. Kuntze }\end{array}$ & seemannoside $A$ (176) & $\begin{array}{l}\text { lisianthioside (177), seemannoside } \\
\left.\text { B (178), (4Z,4a } R^{*}, 12 Z, 12 \mathrm{a} R^{*}\right) \text { - } \\
\text { 4,12-Diethylidene-4,4a,5,6,12, } \\
\text { I2a,13,14-octahiydro-3H, 8H, 11H, } \\
\text { 16H, dipyrano[3,4-c:3',4'-i] [1, 7]di- } \\
\text { oxacyclododecin-3,8,11,I6-tetrone } \\
\text { (179) }\end{array}$ & AF: $C c(179)$ & [60] \\
\hline Lamiaceae & $\begin{array}{l}\text { Cornutia grandifolia var. } \\
\text { intermedia }\end{array}$ & cornutine C-L (180-189) & & AP: $P f(180,181)$ & [61] \\
\hline Lauraceae & $\begin{array}{l}\text { Nectandra lineata (Kunth.) } \\
\text { Rohwer }\end{array}$ & $\begin{array}{l}\text { 3'-methoxy-3,4-methy- } \\
\text { lenedioxy-4',7-epoxy-9- } \\
\text { nor-8,5'-neolignan-9'-acet- } \\
\text { oxy (190) }\end{array}$ & $\begin{array}{l}\text { 3'-methoxy-3,4-methylenedioxy- } \\
\text { 4'-7-epoxy-9-nor-8,5'-neolignan- } \\
\text { 7,8'-diene (191) }\end{array}$ & AT: $T c(190,191)$ & [62] \\
\hline Lythraceae & $\begin{array}{l}\text { Adenaria floribunda } \\
\text { Kunth. }\end{array}$ & adenaflorin A-D (192-195) & & $\begin{array}{l}\text { AC: MCF-7, H-460, } \\
\text { SF-268 (192) }\end{array}$ & [63] \\
\hline Malpighaceae & Hiraea reclinata Jacq. & & $\begin{array}{l}\text { kaempferol 3-O-(6"'-galloyl)- } \beta \text { - } \\
\text { D-galactopyranoside (196), hyperin } \\
\text { 6"-gallate (197), 1,3,4,5-tetra- } \\
\text { galloylquinic acid (198), vitexin } \\
\text { 2"-rhamnoside (199), isovitexin } \\
\text { 2"-rhamnoside (200), orientin } \\
\text { 2"-rhamnoside (201), isoorientin } \\
\text { 2"'-rhamnoside (202) }\end{array}$ & HIV-P: (198) & [64] \\
\hline \multirow[t]{2}{*}{ Malvaceae } & Apeiba tibourbou Aubl. & & rosmarinic acid (203) & IC: (203) & [65] \\
\hline & Guazuma ulmifolia Lam. & & $\begin{array}{l}\text { (-)-epicatechin (204), procianidina } \\
\text { B2 (205), procianidina B5 (206), } \\
\text { procianidina C1 (207) }\end{array}$ & 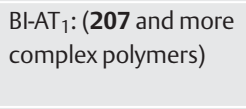 & [66] \\
\hline Melastomataceae & Clidemia sericea D. Don & $\begin{array}{l}2^{\prime \prime}, 6^{\prime \prime}-\text {-Odigalloylvitexin } \\
\text { (208) }\end{array}$ & $\begin{array}{l}\text { isovitexin (209), } 2^{\prime \prime} \text {-O-galloylvitexin } \\
\text { (210), rutin (211), vitexin (212) }\end{array}$ & AP: $P f(208,210)$ & [33] \\
\hline
\end{tabular}


Table 2 Chemistry and biological activity of selected Panamanian plants. (continued)

\begin{tabular}{|c|c|c|c|c|c|}
\hline Family & Species & New compounds & Known compounds & Biological activity & Ref. \\
\hline & $\begin{array}{l}\text { Henriettella fascicularis (Sw.) } \\
\text { C. Wright }\end{array}$ & $\begin{array}{l}\text { 4',5,7-trihydroxy-6,8- } \\
\text { dimethylisoflavone (213), } \\
\text { ácido (2E, 6S)-6- } \\
{[(1 R, 5 Z, 3 \mathrm{a} S, 9 R, 10 Z, 12 \mathrm{a} R)-} \\
\text { 1,2,3,3a,4,7,8,9,12,12a- } \\
\text { decahydro-9-hydroxy- } \\
\text { 3a,6,10-trimethylcyclo- } \\
\text { pentanocycloundecen-1- } \\
\text { yl]-2-methylhept-2-enoic } \\
\text { acid (214) }\end{array}$ & $\begin{array}{l}\text { lichexanthone (215), (-)-pinoresi- } \\
\text { nol (216), betulinic acid (217), } \\
\text { palmitic acid (218), } \beta \text {-sitosterol } \\
\text { (219) }\end{array}$ & AE: (213) & {$[67]$} \\
\hline Myrtaceae & $\begin{array}{l}\text { Calycolpus } \\
\text { warzewiczianus } \\
\text { O. Berg }\end{array}$ & $\begin{array}{l}\text { myricetin-3-O- } \alpha-\mathrm{-}-3^{\prime \prime} \text { - } \\
\text { acetylarabinofuranoside } \\
(220), \text { myricetin-3-O- } \alpha \text {-L- } \\
3^{\prime \prime}-5^{\prime \prime} \text {-diacetylarabinofu- } \\
\text { ranoside (221), and 5-gal- } \\
\text { loylquercetin-3-O- } \alpha \text {---arabi- } \\
\text { nofuranoside (222) }\end{array}$ & $\begin{array}{l}\text { myricetin-3-O- } \alpha \text {---arabinofurano- } \\
\text { side (223), (-)-epicatechin (224) }\end{array}$ & AP: $P f(222)$ & {$[68]$} \\
\hline \multirow[t]{2}{*}{ Siparunaceae } & $\begin{array}{l}\text { Siparuna thecaphora } \\
\text { (Poepp. \& Endl.) } \\
\text { A. DC. = Siparuna } \\
\text { andina (Tul.) A. DC. }\end{array}$ & $\begin{array}{l}\text { sipandinolide (225), } \\
(-) \text {-cis-3-acetoxy-4',5,7- } \\
\text { trihydroxyflavanone (226) }\end{array}$ & & AP: $P f(226)$ & [69] \\
\hline & $\begin{array}{l}\text { Siparuna pauciflora } \\
\text { (Beurl.) A. DC. }\end{array}$ & $\begin{array}{l}\text { sipaucin A (227), B (228), } \\
\text { and C (229) }\end{array}$ & $\begin{array}{l}\text { nor-boldine (230), boldine (231), } \\
\text { laurotetanine (232), N-methyl- } \\
\text { laurotetanine (233) }\end{array}$ & AP: $P f(230)$ & [70] \\
\hline Papaveraceae & Bocconia frutescens $\mathrm{L}$. & & $\begin{array}{l}\text { sanguinarine (234), chelirubine } \\
\text { (235), chelerythrine (236), macar- } \\
\text { pine (237), chelidonin (238), ber- } \\
\text { berine (239), allocryptopine (240), } \\
\text { protopine (241), (-)-isocoripalmine } \\
\text { (242), coptisine (243), aescouler- } \\
\text { ine (244), (-)-cis-N-methyl-cana- } \\
\text { dine (245) }\end{array}$ & 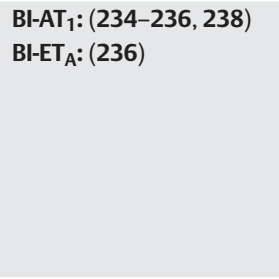 & [71] \\
\hline \multirow[t]{4}{*}{ Piperaceae } & Piper darienense C. DC. & & pipercallosine (246) & Local anesthetic (246) & [72] \\
\hline & Piper dilatatum Rich. & taboganic acid (247) & $\begin{array}{l}\text { methyl taboganate (248), 2,2-di- } \\
\text { methyl-6-carboxychroman-4-one } \\
\text { methyl ester (249), 2,2-dimethyl-3- } \\
\text { hydroxy-6-carboxychromane meth- } \\
\text { yl ester (250), methyl 3-(2'-hy- } \\
\text { droxy-3'-methyl-3'-butenyl)-4-hy- } \\
\text { droxy-benzoate (251), 2,2-dimeth- } \\
\text { yl-6-carboxychromene methyl ester } \\
\text { (252), flavokawin (253), alpinetin } \\
\text { chalcone (254), 2'-hydroxy-3',4',6'- } \\
\text { trimethoxy-chalcone (255) }\end{array}$ & AF: Cc (247-250) & [73] \\
\hline & Piper fimbriulatum C. DC. & $\begin{array}{l}\text { 3,4,5'-trimethoxy-3',4'- } \\
\text { methylenedioxy-7,9':7',9 } \\
\text { diepoxylignan }(\mathbf{2 5 6})\end{array}$ & $\begin{array}{l}\text { 7'-epi-sesartemin (257) and dia- } \\
\text { yangambin (258), 5-hydroxy-7,4'- } \\
\text { dimethoxyflavone (259), } \beta \text {-caryo- } \\
\text { phyllene (260), germacrene (261), } \\
\text { linalol (262), linalol acetate (263) }\end{array}$ & $\begin{array}{l}\text { LC: } A a(257) \\
\text { AP: } P f(257) \\
\text { Al, IS: (257) }\end{array}$ & {$[74-76$} \\
\hline & $\begin{array}{l}\text { Piper multiplinervium C. } \\
\text { DC. }\end{array}$ & $\begin{array}{l}\text { 3-farnesyl-2-hydroxy ben- } \\
\text { zoic acid (264) }\end{array}$ & & $\begin{array}{l}\text { AB: } H p ; S a, E c, K p, M s, P a \\
C a(264)\end{array}$ & [77] \\
\hline Polygalaceae & $\begin{array}{l}\text { Monnina sylvatica } \\
\text { Schltdl. \& Cham. }\end{array}$ & $\begin{array}{l}\text { 3'-Hydroxy-5-methoxy-3,4- } \\
\text { methyilenedioxybiphenyl } \\
(\mathbf{2 6 5}), 3^{\prime} \text {-hydroxy-5,5'-di- } \\
\text { methoxy-3,4-methylene- } \\
\text { dioxybiphenyl }(\mathbf{2 6 6}) \text {, } \\
\text { kaempferol 3-O- } \beta \text {-D-gluco- } \\
\text { syl-( }(1 \rightarrow 2) \text {-O-[ } \alpha \text {---rhamno- } \\
\text { syl }(1 \rightarrow 6)]-\beta \text {-D- } \\
\text { galactoside }(\mathbf{2 6 7})\end{array}$ & 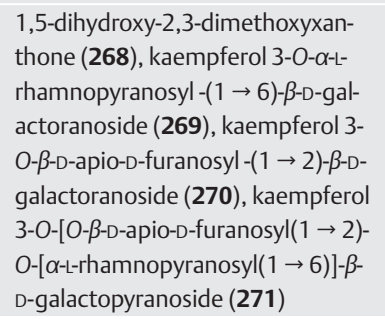 & $\begin{array}{l}\text { AF: } C c(265,266), \\
\operatorname{Sc}(265,266), \\
\operatorname{Af}(265), \operatorname{Tm}(265) \\
\text { TBS: }(265,266)\end{array}$ & {$[78,79]$} \\
\hline
\end{tabular}


Table 2 Chemistry and biological activity of selected Panamanian plants. (continued)

\begin{tabular}{|c|c|c|c|c|c|}
\hline Family & Species & New compounds & Known compounds & Biological activity & Ref. \\
\hline Polygonaceae & $\begin{array}{l}\text { Triplaris cumingiana } \\
\text { Fisch. \& C. A. Mey. ex } \\
\text { Mey. }\end{array}$ & $\begin{array}{l}\text { 2-(3,4-dihydroxyphenyl)- } \\
\text { 5,7-dihydroxy-4-oxo-4H- } \\
\text { chromen-3-yl-4,6-bis-O- } \beta \text { - } \\
\text { D-(3,4,5-trihydroxybenzo- } \\
\text { yl) glucopyranoside (272), } \\
\text { 5,7-dihydroxy-2-(4-hy- } \\
\text { droxyphenyl)-4-oxo-4H- } \\
\text { chromen-3-yl-5-O- } \alpha \text {-- } \\
\text { (3,4,5-trihydroxybenzoyl) } \\
\text { arabinofuranoside (273), } \\
\text { 2-hydroxy-4-O- } \alpha-\text {--(3,5,7- } \\
\text { trihydroxy-4-oxo-4H-chro- } \\
\text { men-2-yl)phenylarabino- } \\
\text { furanoside (274) }\end{array}$ & $\begin{array}{l}\text { quercetin 3-O- } \alpha-\mathrm{-}-\left(5^{\prime \prime}-\mathrm{O} \text {-galloyl)ara- }\right. \\
\text { binofuranoside (275), quercetin 3- } \\
\text { O- } \beta \text {-D- }\left(6^{\prime \prime}-O-\text {-galloyl)glucopyrano- }\right. \\
\text { side (276) }\end{array}$ & $\begin{array}{l}\text { AC: H-460 (272, 274, } \\
\text { 275), MCF-7 (273-275), } \\
\text { SF-268 (275) }\end{array}$ & [80] \\
\hline \multirow[t]{3}{*}{ Rubiaceae } & $\begin{array}{l}\text { Coutarea hexandra } \\
\text { (Jacq.) K. Schum. }\end{array}$ & $\begin{array}{l}\text { 5-O- } \beta \text {-D-glucopyranosyl-4- } \\
\text { (4-hydroxyphenyl)-7-me- } \\
\text { thoxy-2H-chromen-2-one } \\
\text { (277) }\end{array}$ & $\begin{array}{l}\text { coumarin, 5-O- } \beta \text {-D-galactopyrano- } \\
\text { syl-4-(4-hydroxyphenyl)-7-me- } \\
\text { thoxy-2H-chromen-2-one (278), } \\
\text { 23,24-dihydrocucurbitacin F (279), } \\
\text { 23,24-dihydro-25-acetylcucurbita- } \\
\text { cin F (280) and 2-O- } \beta \text {-D-glucopyra- } \\
\text { nosyl-23,24-dihydrocucurbitacin F } \\
\text { (281) }\end{array}$ & $\begin{array}{l}\text { AC: MCF-7, H-460, } \\
\text { SF-268 (279, 281) }\end{array}$ & [81] \\
\hline & $\begin{array}{l}\text { Pogonopus speciosus } \\
\text { (Jacq.) K. Schum. }\end{array}$ & $\begin{array}{l}1^{\prime}, 2^{\prime}, 3^{\prime}, 4^{\prime} \text {-tetradehydrotu- } \\
\text { bulosine ( } 282 \text { ) }\end{array}$ & $\begin{array}{l}\text { tubulosine (283), psychotrine } \\
\text { (284) }\end{array}$ & AC: (283) & [82] \\
\hline & $\begin{array}{l}\text { Notopleura } \\
\text { camponutans (Dwyer \& } \\
\text { M. V. Hayden) C. M. } \\
\text { Taylor = Notopleura cam- } \\
\text { ponutans (Dwyer \& M. V. } \\
\text { Hayden) C. M. Taylor }\end{array}$ & $\begin{array}{l}\text { 1-hydroxybenzoisochro- } \\
\text { manquinone (284) }\end{array}$ & $\begin{array}{l}\text { benz }[\mathrm{g}] \text { isoquinoline-5,10-dione } \\
\text { (285) }\end{array}$ & $\begin{array}{l}\text { TBS, KB: }(284,285) \\
\text { AP: } P f(284,285)\end{array}$ & [83] \\
\hline Selaginellaceae & $\begin{array}{l}\text { Selaginella willdenowii } \\
\text { (Desv. Ex Poir) Baker }\end{array}$ & $\begin{array}{l}2^{\prime \prime}, 3^{\prime \prime} \text {-dihydroisocrypto- } \\
\text { merin (286) }\end{array}$ & $\begin{array}{l}4^{\prime}, 7^{\prime \prime} \text {-di-O-methylamentoflavone } \\
\text { (287), isocryptomerin (288), bilo- } \\
\text { betin (289), } 7^{\prime \prime}-O \text {-methylrobusta- } \\
\text { flavone (290), amentoflavone } \\
\text { (291), robustaflavone (292) }\end{array}$ & $\begin{array}{l}\text { AC: BC1, U373 } \\
(\mathbf{2 8 7}, \mathbf{2 8 8}, \mathbf{2 9 0}), \\
\text { HT-1080, Lu1, Col2, KB, } \\
\text { LNCaP, ZR-75-1 (288, } \\
\text { 290), KB-V+ (287, 288), } \\
\text { KB-V-(288) }\end{array}$ & [84] \\
\hline Simaroubaceae & Simarouba amara Aubl. & & $\begin{array}{l}\text { ailanthinone (293), 2-acetylglau- } \\
\text { carubinone (294), holacanthone } \\
\text { (295), glaucarubinone (296) }\end{array}$ & AP: $P f, P b(295)$ & [85] \\
\hline Solanaceae & $\begin{array}{l}\text { Witheringia solanace } \\
\text { L'Her. }\end{array}$ & & $\begin{array}{l}\text { physalins B (297), F (298), and D } \\
\text { (299), }\end{array}$ & NF-kB: $(297,298)$ & [86] \\
\hline
\end{tabular}

DPPH: radical-scavenging activity of 1,1-diphenyl-2-picrylhydrazine; ALP: antioxidant activity in alkaline phosphatase test; HIV-1 RT: human immunodeficiency virus reverse transcriptase assay; HIV-P: cellular protection against HIV infection; AE: antiestrogenic activity; AB: antibacterial activity; AC: anticancer; Al: anti-inflammatory; AH: antihypertensive; IS: immunosuppressive; AF: antifungal activity; AP: antiplasmodial; AT: antitrypanosomal; L: leishmanicidal; LC: larvicidal; MC: molluscicidal; TBS: toxic to brine shrimp; BI-AT1: binding inhibition on angiotensin II $\mathrm{AT}_{1}$ receptor; BI-ETA: binding inhibition on endothelin $\mathrm{ET}_{\mathrm{A}}$ receptor; Bs: Bacillus subtilis; Ms: Mycobacterium smegmatis; Hp: Helicobacter pylori; Sa: Staphylococcus aureus; Ec: Escherichia coli; Kp: Klebsiella pneumonia; Ms: Mycobacterium smegmatis; Pa: Pseudomonas aeruginosa; Ca: Candida albicans; Ef: Epidermophyton floccosum; Sc: Saccharomyces cerevisiae; Af: Aspergillus fumigatus; Tm: Trichophyton mentagrophytes; Mg: Microsporum gypseum; Sg: Salmonella gallinarum; Cc: Cladosporium cucumerinum; Pf: Plasmodium falciparum; Pb: Plasmodium berghei; Tc: Trypanosoma cruzi; Lm: Leishmania mexicana; Lp: Leishmania panamensis; Aa: Aedes aegypti; Bg: Biomphalaria glabrata; MCF-7: breast human cancer; H-460: lung human cancer; SF-268: CNS human cancer; KB: oral epidermoid carcinoma; BC1: breast cancer; HT-1080: fibrosarcoma; Lu1: lung cancer; Col2: colon cancer; KB-V+: drug-resistant KB in presence of vinblastin; KB-V-: drug resistant KB in absence of vinblastin; LNCaP: hormone-dependent prostate cancer; ZR-75-1: hormone dependent breast cancer; U373: glioblastoma; NF-KB: NF-KB inhibition; IC: inhibition of complement

\section{Acknowledgements}

$\nabla$

Thanks are due to the Organization of American States, National Secretariat for Science, Technology and Innovation of Panama, Interamerican Development Bank, European Union, Iberoamerican Program for Science and Technology for Development (CYTED), Alexander von Humboldt Stiftung, Fundación Natura, International Cooperative Biodiversity Groups (NIH, AID) for support. Thanks are also due to Kurt Hostettmann, Arnold Vlietinck, Luc Pieters, Patrick Vanderheyden, Georges Vauquelin, Arturo San Feliciano, Phyllis Coley, Finn Sandberg, Jose Luis López, Eckert Eich,
Kristina Jennet-Siems, Hans Achenbach, Pablo Solís, Dionisio Olmedo, Angela Calderon, Masao Hattori, Late Antonio González, Hans Becker, Erik Spörle, and Mireya Correa for collaborating. We are grateful to Alex Espinosa for revising the botanical names of the plants and the National Environment Authority of Panama for collection and export permits.

\section{Conflict of Interest \\ $\nabla$}

The authors have no conflict of interest to report. 


\section{References}

1 Newman DJ, Cragg GM. Natural products as sources of new drugs over the last 25 years. J Nat Prod 2007; 70: 461-477

2 Schuster $B G$. A new integrated program for natural product development and the value of an ethnomedical approach. J Altern Complement Med 2001; 7: 61-72

3 Correa MD, Galdames C, Stapf MS. Catálogo de plantas vasculares de Panamá. Panama: Editora Novo Art, SA; 2004: 600

4 Anonymous. New map of biodiversity hot spot aids targeting of conservation efforts. Diversity 1997; 13: 27-29

5 Bartholt W, Lauer W, Placke A. Global distribution of species in vascular plants. In: von Troll CB, editor. Erdkunde. Archiv für Wissenschaftliche Geographie. Kelve: Boss Verlag; 1996: 317-327

6 Gupta MP, Marston A, Hostettman K. Bioactive compounds from Panamanian plants. In: Hostettmann K, Gupta MP, Marston A, editors. Chemistry, biological and pharmacological properties of medicinal plants from the Americas. The Netherlands: Harwood Academic Publishers; 1999: 143-159

7 Gupta MP. Panamanian flora: source of bioactive compounds. In: Hostettmann K, Marston A, Maillard M, Hamburger M, editors. Phytochemistry of plants used in traditional medicine. Oxford: Oxford Science Publications, Clarendon Press; 1995: 359-398

8 Calderón AI, Romero LI, Ortega-Barría E, Solís PN, Zacchino S, Gimenez A, Pinzón R, Cáceres A, Tamayo G, Guerra C, Espinosa A, Correa M, Gupta MP. Screening of Latin American plants for antiparasitic activities against malaria, Chagas disease, and leishmaniasis. Pharm Biol 2010; 48: 545-553

9 Calderón AI, Cubilla M, Espinosa A, Gupta MP. Screening of plants of Amaryllidaceae and related families from Panama as sources of acetylcholinesterase inhibitors. Pharm Biol 2010; 48: 988-993

10 Caballero-George C, Vanderheyden PM, Solis PN, Pieters L, Shahat AA, Gupta MP, Vauquelin G, Vlietinck AJ. Biological screening of selected medicinal Panamanian plants by radioligand-binding techniques. Phytomedicine 2001; 8: 59-70

11 National Science Strategic Plan 2010-2014. Available at http://www. senacyt.gob.pa/doc/PENCYT_completo_Gabinete.pdf. Accessed March 18,2011

12 Gupta MP, Arias T, Correa M. Ethnopharmacognostic observations on Panamanian medicinal plants. Part I. Quart J Crude Drug Res 1979; 17: $115-130$

13 Población Indígena Panameña. Available at http://panama.unfpa.org/ poblacion-panama. Accessed March 18, 2011

14 Gupta MP, Correa MD, Solis PN, Jones A, Galdames C, Guionneau-Sinclair F. Medicinal plant inventory of Kuna Indians: Part I. J Ethnopharmacol 1993; 40: 77-109

15 Joly LG, Guerra S, Septimo R, Solis PN, Correa M, Gupta MP, Levy S, Perera $P$, Sandberg $F$. Ethnobotanical inventory of medicinal plants used by the Guaymi Indians in Western Panama. Part II. J Ethnopharmacol 1990; 28: 191-206

16 Joly LG, Guerra S, Septimo R, Solis PN, Correa M, Gupta MP, Levy S, Sandberg $F$. Ethnobotanical inventory of medicinal plants used by the Guaymi Indians in Western Panama. Part I. J Ethnopharmacol 1987; 20: $145-171$

17 Gupta MP, Solís PN, Calderón AI, Guionneau-Sinclair F, Correa M, Galdames C, Guerra C, Espinosa A, Alvenda GI, Robles G, Ocampo R. Medical ethnobotany of the Teribes of Bocas del Toro, Panama. J Ethnopharmacol 2005; 96: 389-401

18 Convention on Biological Diversity. Available at http://www.cbd.int/ convention/text/. Accessed March 23, 2011

19 Integrating Intellectual Property Rights and Development of Policy. Commission on Intellectual Property Rights. Available at http://www. iprcommission.org/papers/pdfs/final_report/CIPRfullfinal.pdf. Accessed March 23, 2011

20 Decreto Ejecutivo No. 257 de 17 Octubre de 2006, que reglamenta el artículo 71 de la ley 41 de 1 julio de 1998, General de Ambiente. Available at http://www.wipo.int/clea/docs_new/pdf/es/pa/pa025es.pdf. Accessed March 23, 2011

21 Cragg GM, Newman DJ. Nature as source of medicines; novel drugs from nature; screening for antitumor activity. In: Mander L, Lui HW, editors. Comprehensive natural products II chemistry and biology, vol. 3. Oxford: Elsevier; 2010: 135-175

22 Solis PN, Wright CW, Anderson MM, Gupta MP, Phillipson JD. A microwell cytotoxicity assay using Artemia salina (brine shrimp). Planta Med 1993; 59: 250-252
23 Calderón AI, Terreaux C, Gupta MP, Hostettmann K. In vitro cytotoxicity of 11 Panamanian plants. Fitoterapia 2003; 74: 378-383

24 Gupta MP, Solís PB, Miranda C, Montenegro O, Martínez R, Varela LA, Correa MA. Antimicrobial activity in medicinal plants of Panama. Rev Med Panama 1988; 13: 79-84

25 Rahalison L, Hamburger M, Hostettman K, Monod M, Frenk E, Gupta MP, Santana AI, Gonzalez AG. Screening of antifungal activity of Panamanian plants. Int J Pharmacognosy 1993; 31: 68-76

26 Gupta MP, Monge A, Karikas GA, Lopez de Cerain A, Solis PN, de Leon E, Trujillo M, Suarez O, Wilson F, Montenegro G, Noriega Y, Santana AI, Correa $M$, Sanchez $C$. Screening of Panamanian medicinal plants for brine shrimp toxicity, crown gall tumor inhibition, cytotoxicity and DNA intercallation. Int J Pharmacognosy 1996; 34: 19-27

27 Matsuse IT, Lim YA, Hattori M, Correa M, Gupta MP. A search for anti-viral properties in Panamanian medicinal plants. The effects on HIV and its essential enzymes. J Ethnopharmacol 1999; 64: 15-22

28 Corbett Y, Herrera L, Gonzalez J, Cubilla L, Capson TL, Coley PD, Kursar TA, Romero LI, Ortega-Barria E. A novel DNA-based microfluorimetric method to evaluate antimalarial drug activity. Am J Trop Med Hyg 2004; 70: 119-124

29 Segura L, Vila R, Gupta MP, Espósito-Avella M, Adzet T, Cañigueral S. Antiinflammatory activity of Anthurium cerrocampanense croat in rats and mice. J Ethnopharmacol 1998; 61: 243-248

30 Jacobo-Herrera NJ, Bremner P, Marquez N, Gupta MP, Gibbons S, Muñoz E, Heinrich $M$. Physalins from Witheringia solanacea as modulators of the NF-kappaB cascade. J Nat Prod 2006; 69: 328-331

31 Gupta MP, Solis NG, Avella ME, Sanchez C. Hypoglycemic activity of Neurolaena lobata (L.) R. BR. J Ethnopharmacol 1984; 10: 323-327

32 Ioset JR, Marston A, Gupta MP, Hostettmann K. A methylflavan with free radical scavenging properties from Pancratium littorale. Fitoterapia 2001; 72: 35-39

33 Montenegro H, González J, Ortega-Barria E, Cubilla-Rios L. Antiprotozoal activity of flavonoid glycosides isolated from Clidemia sericea and Mosquitoxylon jamaicense. Pharm Biol 2007; 45: 376-380

34 Ceplenaeu F, Ohtani K, Hamburger M, Gupta MP, Solis P, Hostettmann K. Novel acetogenins from the leaves of Annona purpurea. Helv Chim Acta 1993; 76: 1379-1387

35 Wang ZW, Ma WW, McLaughlin JL. 2,4,5-trimethoxystyrene, a bioactive component of the bark of Duguetia panamensis. J Nat Prod 1988; 51: 382-384

36 Montenegro H, Gutierrez M, Romero L, Ortega-Barria E, Capson T, Cubilla Rios $L$. Aporphine alkaloids from Guatteria spp. with leishmanicidal activity. Planta Med 2003; 69: 677-679

37 Correa JE, Ríos CH, del Rosario Castillo A, Romero LI, Ortega-Barría E, Coley PD, Kursar TA, Heller MV, Gerwick WH, Rios LC. Minor alkaloids from Guatteria dumetorum with antileishmanial activity. Planta Med 2006; 72: $270-272$

38 Achenbach H, Löwel M, Waibel R, Gupta M, Solis P. New Lignan Glucosides from Stemmadenia minima. Planta Med 1992; 58: 270-272

39 Gupta MP, Alvarez D, Solis PN, Löwel M, Achenbach H. Phytochemical and biological study of Slemmadenia minima. Planta Med 1991; 57: 502-503

40 Rahalison L, Benathan M, Monod M, Frenk E, Gupta MP, Solis PN, Fuzzati $N$, Hostettmann $K$. Antifungal principles of Baccharis pedunculata. Planta Med 1995; 61: 360-362

41 Martin F, Hay AE, Cressend D, Reist M, Vivas L, Gupta MP, Carrupt PA Hostettmann $K$. Antioxidant C-glucosylxanthones from the leaves of Arrabidaea patellifera. J Nat Prod 2008; 71: 1887-1890

42 Martin F, Hay AE, Quinteros Condoretty VR, Cressend D, Reist M, Gupta MP, Carrupt PA, Hostettmann K. Antioxidant phenylethanoid glycosides and a neolignan from Jacaranda caucana. J Nat Prod 2009; 72: 852-856

43 Ioset JR, Marston A, Gupta MP, Hostettmann K. Antifungal and larvicidal compounds from the root bark of Cordia alliodora. J Nat Prod 2000; 63: 424-426

44 Ioset JR, Marston A, Gupta MP, Hostettmann K. Antifungal and larvicidal meroterpenoid naphthoquinones and a naphthoxirene from the roots of Cordia linnaei. Phytochemistry 1998; 47: 729-734

45 Lim Y, Kida H, Miyaji M, Kusumoto I, Miyashiro H, Hattori M, Shimotohno K, Gupta MP, Correa M. Inhibitory effect of some Panamanian plants on human immunodeficiency viral reverse transcriptase and protease. J Tradit Med 1997; 14: 54-58

46 Matsuse IT, Lim YA, Hattori M, Correa M, Gupta MP. A search for anti-viral properties in Panamanian medicinal plants. The effects on HIV and its essential enzymes. J Ethnopharmacol 1999; 64: 15-22 
47 Ioset JR, Marston A, Gupta MP, Hostettmann K. Antifungal xanthones from roots of Marila laxiflora. Pharm Biol 1998; 36: 103-106

48 López-Pérez JL, Olmedo D, del Olmo E, Vásquez Y, Solís PN, Gupta MP, San Feliciano A. Cytotoxic 4-phenylcoumarins from the leaves of Marila pluricostata. J Nat Prod 2005; 6: 369-373

49 Olmedo DA, López-Pérez JL, del Olmo E, Vásquez Y, San Feliciano A, Gupta $M P$. A new cytotoxic friedelane acid-pluricostatic acid-and other compounds from the leaves of Marila pluricostata. Molecules 2008; 13 : 2915-2924

50 Pecchio M, Solís PN, López-Pérez JL, Vasquez Y, Rodríguez N, Olmedo D, Correa M, San Feliciano A, Gupta MP. Cytotoxic and antimicrobial benzophenones from the leaves of Tovomita longifolia. J Nat Prod 2006; 69: 410-413

51 Hussein AA, Bozzi B, Correa M, Capson TL, Kursar TA, Coley PD, Solis PN, Gupta MP. Bioactive constituents from three Vismia species. J Nat Prod 2003; 66: 858-860

52 Hussein AA, Olmedo DA, Vasquez Y, Coley PD, Solis PN, Gupta MP. New cytotoxic cinnamic acid derivatives from leaves of Bonamia trichantha. Rev Latinoamer Quím 2005; 32: 90-96

53 Rodriguez N, Vasquez Y, Hussein AA, Coley PD, Solis PN, Gupta MP. Cytotoxic cucurbitacin constituents from Sloanea zuliaensis. J Nat Prod 2003; 66: 1515-1516

54 Kraft C, Jenett-Siems K, Siems K, Gupta MP, Bienzle U, Eich E. Antiplasmodial activity of isoflavones from Andira inermis. J Ethnopharmacol 2000; 73: 131-135

55 Maillard M, Hamburger M, Gupta MP, Hostettmann K. An antifungal isoflavanone and a structure revision of a flavanone from Erythrina berteroana. Planta Med 1989; 55: 281-282

56 Maillard M, Gupta MP, Hostettmann K. A new antifungal prenylated flavanone from Erythrina berteroana. Planta Med 1987; 53: 563-564

57 Ioset JR, Marston A, Gupta MP, Hostettmann K. Five new prenylated stilbenes from the root bark of Lonchocarpus chiricanus. J Nat Prod 2001; 64: 710-715

58 Mendoza DT, Ureña González LD, Ortega-Barría E, Capson TL, Rios LC. Five new cassane diterpenes from Myrospermum frutescens with activity against Trypanosoma cruzi. J Nat Prod 2003; 66: 928-932

59 Borel C, Gupta MP, Hostettmann K. Moluscicidal saponins from Swartzia simplex. Phytochemistry 1987; 26: 2685-2689

60 Rodriguez S, Wolfender JL, Hostettmann K, Stoeckli-Evans H, Gupta MP. Monoterpene dimers from Lisianthius seemannii. Helv Chim Acta 1998; 81: 1393-1403

61 Jenett-Siems K, Köhler I, Kraft C, Siems K, Solis PN, Gupta MP, Bienzle U. Cornutins C-L, neo-clerodane-type diterpenoids from Cornutia grandifolia var. intermedia. Phytochemistry 2003; 64: 797-804

62 Chérigo L, Polanco V, Ortega-Barria E, Heller MV, Capson TL, Rios LC. Antitrypanosomal activity of a novel norlignan purified from Nectandra lineata. Nat Prod Res 2005; 19: 373-377

63 Hussein AA, Barberena I, Capson TL, Kursar TA, Coley PD, Solis PN, Gupta $M P$. New cytotoxic naphthopyrane derivatives from Adenaria floribunda. J Nat Prod 2004; 67: 451-453

64 Hussein AA, Gomez B, Ramos M, Heller M, Coley P, Solis PN, Gupta MP. Constituents of Hiraea reclinata and their anti-HIV activity. Rev Latinoamer Quím 2003; 31: 74-77

65 Lasure A, Van Poel B, Pieters L, Claeys M, Gupta M, Vanden Berghe D, Vlietinck AJ. Complement-inhibiting properties of Apeiba tibourbou. Planta Med 1994; 60: 276-277

66 Caballero-George C, Vanderheyden PM, De Bruyne T, Shahat AA, Van den Heuvel H, Solis PN, Gupta MP, Claeys M, Pieters L, Vauquelin G, Vlietinck AJ. In vitro inhibition of $\left[{ }^{3} \mathrm{H}\right]$-angiotensin II binding on the human $\mathrm{AT}_{1}$ receptor by proanthocyanidins from Guazuma ulmifolia bark. Planta Med 2002; 68: 1066-1071

67 Calderón AI, Terreaux C, Schenk K, Pattison P, Burdette JE, Pezzuto JM, Gupta MP, Hostettmann K. Isolation and structure elucidation of an isoflavone and a sesterterpenoic acid from Henriettella fascicularis. J Nat Prod 2002; 65: 1749-1753
68 Torres-Mendoza D, González J, Ortega-Barría E, Heller MV, Capson TL, McPhail K, Gerwick WH, Cubilla-Rios L. Weakly antimalarial flavonol arabinofuranosides from Calycolpus warszewiczianus. J Nat Prod 2006; 69: 826-828

69 Jenett-Siems K, Siems K, Jakupovic J, Solis PN, Gupta MP, Mockenhaupt FP, Bienzle U, Eich E. Sipandinolide: a butenolide including a novel type of carbon skeleton from Siparuna andina. Planta Med 2000; 66: 384-385

70 Jenett-Siems K, Kraft C, Siems K, Jakupovic J, Solis PN, Gupta MP, Bienzle $U$. Sipaucins A-C, sesquiterpenoids from Siparuna pauciflora. Phytochemistry 2003; 63: 377-381

71 Caballero-George C, Vanderheyden PM, Apers S, Van den Heuvel H, Solis PN, Gupta MP, Claeys M, Pieters L, Vauquelin G, Vlietinck AJ. Inhibitory activity on binding of specific ligands to the human angiotensin II AT (1) and endothelin 1 ET(A) receptors: bioactive benzo[c]phenanthridine alkaloids from the root of Bocconia frutescens. Planta Med 2002; 68: 770-775

72 Rodríguez N, Rodríguez M, Calderón A, San Feliciano A, Solís PN, Gupto MP. Anesthetic activity of pipercallosine isolated from Piper darienense. Rev Latinoamer Quím 2005; 33: 115-120

73 Terreaux C, Gupta MP, Hostettman K. Antifungal benzoic acid derivatives from Piper dilatatum. Phytochemistry 1998; 49: 461-464

74 Solís PN, Olmedo D, Nakamura N, Calderón A, Hattori M, Gupta MP. A new larvicidal lignan from Piper fimbriulatum. Pharm Biol 2005; 43: 378-381

75 De León EJ, Olmedo DA, Solís PN, Gupta MP, Terencio MC. Diayangambin exerts immunosuppressive and anti-inflammatory effects in vitro and in vivo. Planta Med 2002; 68: 1128-1131

76 Mundina M, Vila R, Tomi F, Gupta MP, Adzet T, Casanova J, Cañigueral S. Leaf essential oils of three Panamanian Piper species. Phytochemistry 1998; 47: 1277-1282

77 Rüegg T, Calderón AI, Queiroz EF, Solís PN, Marston A, Rivas F, OrtegaBarría E, Hostettmann K, Gupta MP. 3-Farnesyl-2-hydroxybenzoic acid is a new anti-Helicobacter pylori compound from Piper multiplinervium. J Ethnopharmacol 2006; 103: 461-467

78 Bashir A, Hamburger M, Rahalison L, Monod M, Gupta MP, Solis P, Hostettmann K. Antifungal biphenyls from Monnina sylvatica. Planta Med 1991; 57: 192-193

79 Bashir A, Hamburger M, Gupta MP, Solis PN, Hostettmann K. Flavonol glycosides from Monnina sylvatica. Phytochemistry 1991; 30: 37813784

80 Hussein AA, Barberena I, Correa M, Coley PD, Solis PN, Gupta MP. Cytotoxic flavonol glycosides from Triplaris cumingiana. J Nat Prod 2005; 68: 231-233

81 Olmedo D, Rodríguez N, Vásquez Y, Solís PN, López-Pérez JL, Feliciano AS, Gupta MP. A new coumarin from the fruits of Coutarea hexandra. Nat Prod Res 2007; 21: 625-631

82 Ito A, Lee YH, Chai HB, Gupta MP, Farnsworth NR, Cordell GA, Pezzuto JM, Kinghorn $A D$. 1',2',3',4'-tetradehydrotubulosine, a cytotoxic alkaloid from Pogonopus speciosus. J Nat Prod 1999; 62: 1346-1348

83 Solis PN, Lang'at C, Gupta MP, Kirby GC, Warhusrst DC, Phillipson JD. Bioactive compounds from Psychotria camponutans. Planta Med 1995; 61: $62-65$

84 Silva GL, Chai H, Gupta MP, Farnsworth NR, Cordell GA, Pezzuto JM, Beecher CW, Kinghorn AD. Cytotoxic biflavonoids from Selaginella willdenowii. Phytochemistry 1995; 40: 129-134

85 O'Neill MJ, Bray DH, Boardman P, Wright CW, Phillipson JD, Warhurst DC, Gupta MP, Correya M, Solis P. Plants as sources of antimalarial drugs, Part 6: Activities of Simarouba amara fruits. J Ethnopharmacol 1988; 22: $183-190$

86 Jacobo-Herrera NJ, Bremner P, Marquez N, Gupta MP, Gibbons S, Muñoz E, Heinrich M. Physalins from Witheringia solanacea as modulators of the NF-kappaB cascade. J Nat Prod 2006; 69: 328-331 